\title{
Host Range of Fusarium Dieback and Its Ambrosia Beetle (Coleoptera: Scolytinae) Vector in Southern California
}

\author{
Akif Eskalen, Department of Plant Pathology and Microbiology, Richard Stouthamer, Department of Entomology, Shannon Colleen \\ Lynch, Department of Plant Pathology and Microbiology, and Center for Conservation Biology, Paul F. Rugman-Jones, Department of \\ Entomology, and Mathias Twizeyimana and Alex Gonzalez, Department of Plant Pathology and Microbiology, University of Califor- \\ nia, Riverside, 92521; and Tim Thibault, Huntington Library, Art Collections, and Botanical Gardens, San Marino 91108
}

\begin{abstract}
Eskalen, A., Stouthamer, R., Lynch, S. C., Twizeyimana, M., Gonzalez, A., and Thibault, T. 2013. Host range of Fusarium dieback and its ambrosia beetle (Coleoptera: Scolytinae) vector in southern California. Plant Dis. 97:938-951.

The polyphagous shot hole borer (PSHB) is an invasive ambrosia beetle that forms a symbiosis with a new, as-yet-undescribed Fusarium sp., together causing Fusarium dieback on avocado and other host plants in California and Israel. In California, PSHB was first reported on black locust in 2003 but there were no records of fungal damage until 2012, when a Fusarium sp. was recovered from the tissues of several backyard avocado trees infested with PSHB in Los Angeles County. The aim of this study was to determine the plant host range of the beetle-fungus complex in two heavily infested botanical gardens in Los Angeles County. Of the 335 tree species observed, 207 (62\%), representing 58 plant families, showed signs and symptoms consistent

with attack by PSHB. The Fusarium sp. was recovered from $54 \%$ of the plant species attacked by PSHB, indicated by the presence of the Fusarium sp. at least at the site of the entry hole. Trees attacked by PSHB included 11 species of California natives, 13 agriculturally important species, and many common street trees. Survey results also revealed 19 tree species that function as reproductive hosts for PSHB. Additionally, approximately a quarter of all tree individuals planted along the streets of southern California belong to a species classified as a reproductive host. These data suggest the beetle-disease complex potentially may establish in a variety of plant communities locally and worldwide.
\end{abstract}

Since the beginning of 2012, an emerging plant disease, referred to as Fusarium dieback (FD), has been detected on several host plants in Los Angeles and Orange Counties, CA (7). FD is caused by the combined effects of a tiny $(<2.5 \mathrm{~mm})$ invasive ambrosia beetle (Scolytinae) and its symbiotic fungal partner, a currently undescribed Fusarium sp. $(7,15)$. Adult female beetles burrow into a tree, creating brood galleries beyond the cambium, which are concurrently inoculated with the Fusarium sp. The fungus colonizes the gallery walls and becomes the sole source of food for developing larvae and adult beetles $(7,15)$. The fungus invades the vascular tissue of the tree, blocking water and nutrients from the roots to the rest of the tree, eventually causing branch dieback and tree death (Fig. 1) $(7,15)$.

The specific identity of the beetle is currently unknown. It is morphologically indistinguishable from the tea shot hole borer (TSHB), Euwallacea fornicatus, a serious pest of tea (Camellia sinensis) in Sri Lanka and India (5) (Fig. 2A-C). However, there are significant differences in nuclear and mitochondrial DNA sequences between the California beetles and those from tea plantations in Sri Lanka and other Asian collection sites (P. F. RugmanJones and R. Stouthamer, unpublished data). This indicates that the California beetle is most likely a separate, congeneric species (representative mitochondrial sequences for the two species are available in GenBank [1], accession numbers JX912723-JX912725). Species within the genus Euwallacea have been subject to much revision and synonymization (28); therefore, establishing the exact identity of this beetle is likely to require extensive taxonomic research. Thus, for the purpose of this study, the beetle is simply

Corresponding author: A. Eskalen, E-mail: akif.eskalen@ucr.edu

* The $\boldsymbol{e}$-Xtra logo stands for "electronic extra" and indicates that Figures 1 to 5,7 , and 8 appear in color in the online edition.

Accepted for publication 24 January 2013.

http://dx.doi.org/10.1094/PDIS-11-12-1026-RE

(C) 2013 The American Phytopathological Society referred to as Euwallacea sp. and the common name polyphagous shot hole borer (PSHB) is used.

In California, PSHB was first collected in 2003 from several tree species (20) but there were no reports of fungal damage before 2012. Preliminary survey data in 2012 showed that backyard avocado trees and many other woody plants in the urban landscape, especially castor bean (Ricinus communis), were heavily infested with this beetle-fungus disease complex in Los Angeles and Orange counties (A. Eskalen, unpublished data). Some of the most heavily infested areas in southern California are home to two of its biggest botanical gardens, the Los Angeles (LA) Arboretum and The Huntington Library, Art Collections and Botanical Gardens, which are relatively close to the original 2003 find of the beetle. These botanical gardens harbor a wide range of plant species that represent unique and common ecosystems from all over the world, and contain all the host species planted throughout urban forests in

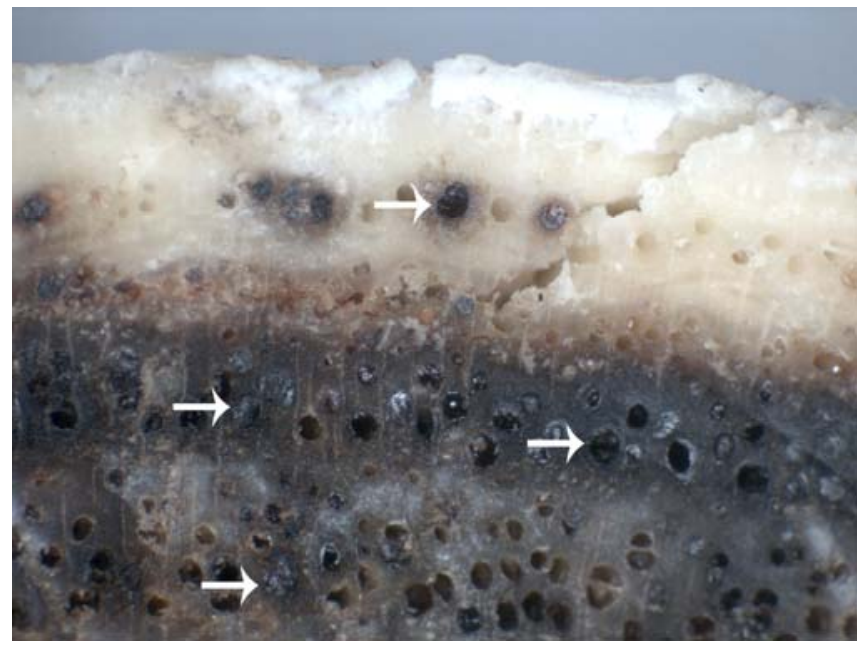

Fig. 1. Wood discoloration and xylem vessel plugging caused by Fusarium sp. on Albizia julibrissin. 
southern California. In addition, the collections receive more consistent and even care as opposed to lands with multiple management strategies. Therefore, the botanical gardens provided a perfect natural laboratory to investigate the plant host range of the beetlefungus complex. An understanding of the host range of the beetlefungus complex is important in determining the present disease distribution and to make predictions about its future distribution.

At present, the beetle-fungus complex appears to be limited to the urban forest. In itself, this will likely present economic problems for households and cities that must bear the cost of removing dead or dying trees. However, the same disease complex was also found in Israel in 2009 in commercial avocado orchards, where it causes significant damage to avocado trees (15). Thus, the presence of FD in urban landscapes throughout southern California is a concern for the California avocado industry because it may eventually spread to commercial avocado groves. Similarly, the wide host range of this beetle may ultimately threaten native trees in the natural forests of southern California.

The objectives of this study were to (i) determine the plant host range of the PSHB and Fusarium sp. and (ii) characterize their symptoms on different host plants.

\section{Materials and Methods}

Host identification and symptom characterization. Trees were visited and identified to species at the LA Arboretum and The Huntington Library, Art Collections and Botanical Gardens in Los Angeles County; specific locations of sampled trees were noted using global positioning system equipment (Garmin 60CSx, 5- to $10-\mathrm{m}$ accuracy). Each botanical garden covers approximately 48 ha and, in general, the species collections housed in each are different. Although the gardens contain a large number of plant species, the criteria in host plant selection included (i) common street trees found in southern California (4), (ii) agriculturally important trees and vines, (iii) species of tree families, and (iv) trees randomly encountered in the search for species outlined using the criteria i-iii.

Signs and symptoms of FD (presence or absence; see Results), severity of beetle colonization $(0=$ none, $1=$ minor $[1$ to 10 entry holes], $2=$ moderate [10 to 30 entry holes], $3=$ [ $>31$ entry holes]), and branch dieback $(0=$ none, $1=$ minor $[1$ to $25 \%$ of branches showed dieback], $2=$ moderate [ 25 to $50 \%$ ], and $3=$ severe $[>50 \%]$ ) were also recorded. For each tree species showing symptoms characteristic of FD (one tree per species), tissue samples were collected by extracting symptomatic tissue from the trunk with a sterilized knife to a depth beyond the cambium. Samples were returned to the laboratory (University of California, Riverside) for morphological and molecular identification of the beetle, the fungus, or both. If visible, beetles were collected and put directly into $95 \%$ ethanol for later molecular identification (see below). The data were analyzed using JMP 9.0.0 statistical software (SAS 2012). Logistic regression was used to determine the presence and absence of Fusarium sp., gumming, and severity of beetle attack as contributing factors to branch dieback $>50 \%$ at $\alpha=0.05$.

The suitability of a tree species to act as a reproductive host for the PSHB was evaluated, where possible, by cutting into trees and confirming the presence of eggs, larvae, pupae, or teneral females or males in the galleries. Trees that did not show signs of heavy infestation by PSHB were not cut to examine and confirm as a reproductive host because species of these trees were limited in number within the botanical gardens' collections.

Fusarium sp. and PSHB confirmation. To carry out fungal isolations, gross contamination was removed before bringing plant samples into a clean area by briefly flaming samples and removing the outer surface with a sterilized paring knife. All samples were then split in half and pieces excised from the leading margin of clean necrotic tissue were plated onto potato dextrose agar (Difco) amended with $100 \mu \mathrm{g}$ of tetracycline (Fisher Scientific). Plates were incubated at $25^{\circ} \mathrm{C}$, and fungal growth was subcultured for identification and long-term storage after 7 to 10 days.

Total genomic DNA was extracted from the mycelium of each isolate using the method described by Cenis (2). Portions of the following four gene fragments were chosen for multilocus sequence typing based on published genetic analyses $(3,16-18,29)$ : the internal transcribed spacer (ITS) region, translation elongation factor 1- $\alpha$ (EF1- $\alpha)$, the second-largest subunit of RNA polymerase $(R P B 2)$, and DNA-directed RNA polymerase subunit 1 (RPB1). Each $25-\mu$ polymerase chain reaction $(\mathrm{PCR})$ mixture contained $19.4 \mu \mathrm{l}$ of PCR-grade water, $2.5 \mu \mathrm{l}$ of standard Taq reaction buffer, $0.5 \mu \mathrm{l}$ of dNTPs $(10 \mathrm{mM}), 0.125 \mu \mathrm{l}$ of NEB Taq DNA polymerase (5,000 U/ml; New England BioLabs), $0.5 \mu \mathrm{l}$ of each primer at 10 $\mathrm{mM}$, and $1.5 \mu \mathrm{l}$ of template DNA. PCR was carried out in a thermal cycler (Bio-Rad Laboratories, Inc.) using published cycling conditions $(14,17,25)$. Amplification products were separated by electrophoresis in $1.5 \%$ agarose gels in 1.0 $\times$ Tris-boric acid-EDTA buffer and visualized under UV light after staining with SYBR Green (Invitrogen). PCR products were purified using an Exo SAP-IT kit (Affymetrix). ITS, EF1- $\alpha$, RPB1, and RPB2 regions were sequenced in both directions at the Institute for Integrative Genome Biology at the University of California, Riverside. Sequences of isolates from the present study were confirmed as Fusarium sp. after a BLAST search comparison in GenBank, which showed they were $100 \%$ homologous to known sequences of isolates of Fusarium sp. from Eskalen et al. and Mendel et al. $(7,15)$. The ITS, EF1- $\alpha$, RPB1, and RPB2 sequences used in this study were deposited into GenBank.

PSHB collection and confirmation. When present, live beetles were collected directly into $95 \%$ ETOH and returned to the laboratory for identification. In the field, beetles were collected from the surface of the trees using forceps. If they were in a gallery, the beetles were first coaxed to the entrance by spraying $95 \%$ ethanol in the gallery and then removed with the forceps (11).

In the laboratory, DNA was extracted from individual beetles by grinding two to three tibia in $2 \mu \mathrm{l}$ of proteinase-K $\left(>600 \mathrm{mAU} \mathrm{ml}^{-1}\right.$, Qiagen) and $35 \mu$ of Chelex 100 resin (Bio-Rad Laboratories) followed by incubation for $1 \mathrm{~h}$ at $55^{\circ} \mathrm{C}$, then for $10 \mathrm{~min}$ at $99^{\circ} \mathrm{C}$. A section of the cytochrome $\mathrm{c}$ oxidase subunit I gene commonly used in barcoding studies (10) was amplified using the primers LCO1490 (5'-GGTCAACAAATCATAAAGATATTGG-3') and
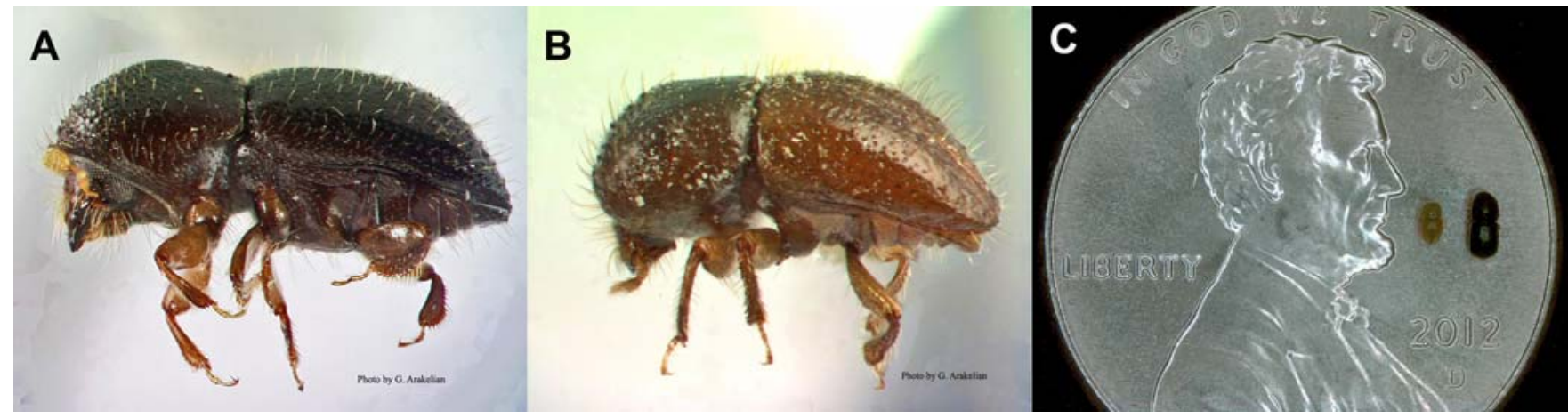

Fig. 2. Polyphagous shot hole borer (PSHB) (Euwallacea sp.): A, female; B, male; C, size comparison of both male (left) and female (right) on a penny. 
Table 1. Hosts of the polyphagous shot hole borer and their susceptibility to Fusarium dieback (FD) ${ }^{\mathrm{a}}$

\begin{tabular}{|c|c|c|c|c|c|c|c|c|}
\hline Family, species ${ }^{\mathbf{b}}$ & Common name & $N^{\mathrm{c}}$ & Crop $^{\mathrm{d}}$ & Origin & $\mathbf{F D}^{\mathbf{e}}$ & $\mathbf{S}$ & $\mathbf{P}$ & $\mathbf{G}$ \\
\hline \multicolumn{9}{|l|}{ Aceraceae s.s. (Sapindaceae s.1.) } \\
\hline *Acer buergerianum & Trident maple & 4 & $\ldots$ & East China, Taiwan & $\mathrm{Y}$ & $\ldots$ & $\ldots$ & $\ldots$ \\
\hline A. davidii & Pere David's maple & $\ldots$ & $\ldots$ & China & $\ldots$ & $\mathrm{Y}$ & $\ldots$ & $\ldots$ \\
\hline A. caudatifolium (syn. A. kawakamii) & Kawakami maple & $\ldots$ & $\ldots$ & Taiwan & $\ldots$ & $\mathrm{Y}$ & $\ldots$ & $\ldots$ \\
\hline *A. macrophyllum & Big leaf maple & $\ldots$ & $\ldots$ & Western North America & $\mathrm{Y}$ & $\mathrm{Y}$ & $\ldots$ & $\ldots$ \\
\hline *A. negundo & Box elder & $\ldots$ & $\ldots$ & North America & $\mathrm{Y}$ & $\mathrm{Y}$ & $\ldots$ & $\ldots$ \\
\hline *A. palmatum 'Bonfire' & Bonfire Japanese maple & $\ldots$ & $\ldots$ & East Asia & $\mathrm{Y}$ & $\ldots$ & $\ldots$ & $\ldots$ \\
\hline *A. paxii & Evergreen maple & 3 & $\ldots$ & Southwest China & $\mathrm{Y}$ & $\mathrm{Y}$ & $\ldots$ & $\ldots$ \\
\hline A. $\times$ freemanii & Freeman maple & $\ldots$ & $\ldots$ & Eastern North America & $\ldots$ & $\mathrm{Y}$ & $\ldots$ & $\ldots$ \\
\hline \multicolumn{9}{|l|}{ Alangiaceae s.s. (Cornaceae s.1.) } \\
\hline Alangium chinensis & $\ldots$ & $\ldots$ & $\ldots$ & Tropical Africa to China & $\mathrm{Y}$ & $\mathrm{Y}$ & $\ldots$ & $\ldots$ \\
\hline \multicolumn{9}{|l|}{ Anacardiaceae } \\
\hline Pistacia chinensis & Chinese pistache & 74 & $\ldots$ & China & $\ldots$ & $\ldots$ & $\ldots$ & $\mathrm{Y}$ \\
\hline Schinus terebinthifolius & Brazilian pepper tree & 333 & $\ldots$ & Brazil & $\mathrm{Y}$ & $\mathrm{Y}$ & $\ldots$ & $\ldots$ \\
\hline \multicolumn{9}{|l|}{ Apocynaceae } \\
\hline Thevetia thevetioides & Yellow oleander & $\ldots$ & $\ldots$ & Mexico & $\ldots$ & $\mathrm{Y}$ & $\ldots$ & $\ldots$ \\
\hline \multicolumn{9}{|l|}{ Aquifoliaceae } \\
\hline Ilex aquifolium & English holly & $\cdots$ & $\ldots$ & $\begin{array}{l}\text { Western and Southern Europe, } \\
\text { Northwest Africa, Southwest Asia }\end{array}$ & $\ldots$ & $\ldots$ & $\ldots$ & \\
\hline *I. cornuta & Chinese holly & $\ldots$ & $\ldots$ & China and Korea & $\mathrm{Y}$ & $\mathrm{Y}$ & $\ldots$ & $\mathrm{Y}$ \\
\hline I. latifolia & Tarajo holly & $\ldots$ & $\ldots$ & Japan and China & $\mathrm{Y}$ & $\mathrm{Y}$ & $\mathrm{Y}$ & $\ldots$ \\
\hline \multicolumn{9}{|l|}{ Araliaceae } \\
\hline Cussonia spicata & Spiked cabbage tree & $\ldots$ & $\ldots$ & South Africa & Y & $\mathrm{Y}$ & $\ldots$ & $\mathrm{Y}$ \\
\hline Fatsia japonica & Japanese aralia & $\ldots$ & $\ldots$ & Japan & $\mathrm{Y}$ & $\mathrm{Y}$ & $\ldots$ & $\mathrm{Y}$ \\
\hline \multicolumn{9}{|l|}{ Arecaceae } \\
\hline Brahea armata & Mexican blue palm & 1 & $\ldots$ & Western Mexico & $\ldots$ & $\ldots$ & $\ldots$ & $\ldots$ \\
\hline Butia capitata & Pindo palm & $\ldots$ & $\ldots$ & Brazil, Uruguay, Argentina & $\ldots$ & $\mathrm{Y}$ & $\ldots$ & $\mathrm{Y}$ \\
\hline Livistona chinensis & Chinese fan palm & $\ldots$ & $\ldots$ & Australia, Southeast Asia & $\mathrm{Y}$ & $\ldots$ & $\ldots$ & $\mathrm{Y}$ \\
\hline Washingtonia filifera & Desert fan palm & 54 & $\cdots$ & Southwest United States, & & & & \\
\hline \multicolumn{9}{|l|}{ Asparagaceae } \\
\hline Draceana draco & Canary islands dragon tree & $\ldots$ & $\ldots$ & $\begin{array}{l}\text { Canary Islands, Cape Verde, Madeira, } \\
\text { Morocco }\end{array}$ & $\ldots$ & $\mathrm{Y}$ & $\ldots$ & $\ldots$ \\
\hline \multicolumn{9}{|c|}{ 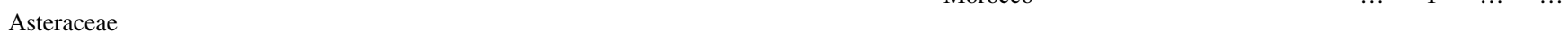 } \\
\hline Verbesina gigantea & Crownbeard & $\ldots$ & $\ldots$ & Tropical America & $\ldots$ & $\ldots$ & $\ldots$ & $\mathrm{Y}$ \\
\hline Betulaceae & & $\ldots$ & $\ldots$ & & & & & \\
\hline Alnus incana & Grey alder & $\ldots$ & $\ldots$ & Europe, Caucasus & $\ldots$ & Y & $\ldots$ & $\mathrm{Y}$ \\
\hline A. rhombifolia & white alder & 72 & $\ldots$ & Western United States & $\ldots$ & $\mathrm{Y}$ & $\ldots$ & $\ldots$ \\
\hline Betula pendula & Silver birch & 12 & $\ldots$ & Europe, Asia Minor & $\mathrm{Y}$ & $\ldots$ & $\ldots$ & $\ldots$ \\
\hline Corylus colurna & Turkish hazelnut & $\ldots$ & Y & Southeast Europe, Asia Minor, & $\mathrm{Y}$ & & & \\
\hline \multicolumn{9}{|l|}{ Bignoniaceae } \\
\hline Catalpa speciosa & Northern catalpa & 8 & $\ldots$ & Central and Eastern United States & Y & Y & $\ldots$ & $\ldots$ \\
\hline Handroanthus impetiginosus & & & & & & & & \\
\hline (syn. Tabebuia impetiginosa) & Brazilwood & $\ldots$ & $\ldots$ & Mexico to South America & $\ldots$ & $\ldots$ & $\mathrm{Y}$ & $\ldots$ \\
\hline \multicolumn{9}{|l|}{ Bombacaceae s.s. (Malvaceae s.1.) } \\
\hline Bombax ceiba & Cotton tree & $\ldots$ & $\ldots$ & India, China, Southeast Asia & $\ldots$ & $\ldots$ & $\ldots$ & $\mathrm{Y}$ \\
\hline Ceiba speciosa & Silk floss tree & 14 & $\ldots$ & Brazil, Argentina & $\mathrm{Y}$ & $\mathrm{Y}$ & $\ldots$ & $\mathrm{Y}$ \\
\hline Pseudobombax ellipticum & Shaving brush tree & $\ldots$ & $\ldots$ & Mexico and Central America & $\ldots$ & $\ldots$ & $\ldots$ & $\mathrm{Y}$ \\
\hline Buddlejaceae & & & & & & & & \\
\hline Nuxia floribunda & Forest elder & $\ldots$ & $\ldots$ & Africa & $\ldots$ & $\ldots$ & $\ldots$ & $\ldots$ \\
\hline Burseraceae & & & & & & & & \\
\hline Bursera hindsiana & $\ldots$ & $\ldots$ & $\ldots$ & Western Mexico & $\ldots$ & $\mathrm{Y}$ & $\ldots$ & $\ldots$ \\
\hline Casuarinaceae & & & & & & & & \\
\hline Casuarina cunninghamiana & She-oak & $\cdots$ & $\cdots$ & Northeast Australia & $\ldots$ & $\mathrm{Y}$ & $\ldots$ & $\ldots$ \\
\hline Cunoniaceae & & & & & & & & \\
\hline Cunonia capensis & Buttetspoon tree & $\ldots$ & $\ldots$ & South Africa & $\ldots$ & $\mathrm{Y}$ & $\ldots$ & $\ldots$ \\
\hline Cornaceae & & & & & & & & \\
\hline Cornus controversa & Giant dogwood & $\ldots$ & $\ldots$ & Himalayas to Japan & $\mathrm{Y}$ & $\ldots$ & $\ldots$ & $\ldots$ \\
\hline Cupressaceae & & & & & & & & \\
\hline Juniperus chinensis & Chinese juniper & 26 & $\ldots$ & Northeast Asia & Y & $\ldots$ & $\ldots$ & $\mathrm{Y}$ \\
\hline J. virginiana & Eastern red-cedar & $\ldots$ & $\ldots$ & Eastern North America & $\ldots$ & $\ldots$ & $\ldots$ & $\mathrm{Y}$ \\
\hline Ebenaceae & & & & & & & & \\
\hline Diospyros kaki & Japanese persimmon & 2 & $\mathrm{Y}$ & Asia & $\ldots$ & $\mathrm{Y}$ & $\ldots$ & $\ldots$ \\
\hline D. lycidioides & $\ldots$ & $\ldots$ & $\mathrm{Y}$ & South Africa & Y & Y & $\ldots$ & $\ldots$ \\
\hline Elaeocarpaceae & & & & & & & & \\
\hline Crinodendron patagua & Lily of the valley tree & $\ldots$ & $\ldots$ & Chile & $\ldots$ & $\mathrm{Y}$ & $\ldots$ & $\ldots$ \\
\hline Ericaceae & & & & & & & & \\
\hline Arbutus unedo & Strawberry tree & $\ldots$ & $\ldots$ & Southwest Ireland to Asia Minor & $\ldots$ & $\mathrm{Y}$ & $\ldots$ & $\ldots$ \\
\hline & & & & & (continu & do & next $p$ & age) \\
\hline
\end{tabular}

a $\mathrm{S}=$ staining, $\mathrm{P}=$ powder, $\mathrm{G}=$ gumming, and $\mathrm{Y}=$ "yes".

b Asterisks: * indicates reproductive hosts and $* *$ indicates critically endangered tree.

${ }^{c}$ Number of times planted along streets of City of Orange (4).

d Agriculturally important crop.

${ }^{\mathrm{e}}$ FD susceptible. 
Table 1. (continued from preceding page)

\begin{tabular}{|c|c|c|c|c|c|c|c|c|}
\hline Family, species ${ }^{b}$ & Common name & $N^{\mathrm{c}}$ & Crop $^{\mathrm{d}}$ & Origin & $\mathbf{F D}^{\mathbf{e}}$ & $\mathbf{S}$ & $\mathbf{P}$ & $\mathbf{G}$ \\
\hline \multicolumn{9}{|l|}{ Euphorbiaceae } \\
\hline Aleurites fordii & Tung oil tree & $\ldots$ & $\ldots$ & Asia & $\ldots$ & $\ldots$ & $\ldots$ & $\mathrm{Y}$ \\
\hline Bischofia javanica & Bishop wood & $\ldots$ & $\ldots$ & Tropical Asia & Y & $\mathrm{Y}$ & $\ldots$ & $\ldots$ \\
\hline Jatropha cf. cinerea & Limberbush & $\ldots$ & $\ldots$ & Arizona, Western Mexico & $\mathrm{Y}$ & $\ldots$ & $\ldots$ & $\ldots$ \\
\hline Manihot esculenta & Cassava & $\ldots$ & $\ldots$ & Brazil & $\ldots$ & $\ldots$ & $\ldots$ & $\ldots$ \\
\hline *Ricinus communis & Castor bean & $\ldots$ & $\ldots$ & Mediterranean basin, East Africa, India & $\mathrm{Y}$ & $\mathrm{Y}$ & $\ldots$ & $\ldots$ \\
\hline Sapium sebiferum & Chinese tallow tree & $\ldots$ & $\ldots$ & China, Japan & $\mathrm{Y}$ & $\mathrm{Y}$ & $\ldots$ & $\ldots$ \\
\hline \multicolumn{9}{|l|}{ Fabaceae } \\
\hline Acacia caven & Roman cassie & $\ldots$ & $\ldots$ & South America & $\ldots$ & $\mathrm{Y}$ & $\ldots$ & $\ldots$ \\
\hline A. stenophylla & Shoestring acacia & $\ldots$ & $\ldots$ & Australia & $\ldots$ & $\ldots$ & $\ldots$ & $\mathrm{Y}$ \\
\hline A. visco & Arca & $\ldots$ & $\ldots$ & Argentina & $\mathrm{Y}$ & $\ldots$ & $\ldots$ & $\ldots$ \\
\hline Albizia kalkora & Kalkora mimosa & $\ldots$ & $\ldots$ & Southern Asia & $\ldots$ & $\mathrm{Y}$ & $\ldots$ & $\ldots$ \\
\hline *A. julibrissin & Silk tree & $\ldots$ & $\ldots$ & Southwest to east Asia & $\mathrm{Y}$ & $\mathrm{Y}$ & $\ldots$ & $\ldots$ \\
\hline Bauhinia $\times$ blakeana & Orchid tree & 1 & $\ldots$ & China & $\mathrm{Y}$ & $\ldots$ & $\ldots$ & $\mathrm{Y}$ \\
\hline B. galpinii & Red orchid bush & $\ldots$ & $\ldots$ & South Africa & $\ldots$ & $\mathrm{Y}$ & $\mathrm{Y}$ & $\ldots$ \\
\hline Calpurnia aurea & $\ldots$ & $\ldots$ & $\ldots$ & Southern India, Africa & $\mathrm{Y}$ & $\mathrm{Y}$ & $\ldots$ & $\mathrm{Y}$ \\
\hline Cassia brewsteri & Cassia pea & $\ldots$ & $\ldots$ & Northeast Australia & $\mathrm{Y}$ & $\mathrm{Y}$ & $\ldots$ & $\mathrm{Y}$ \\
\hline *Castanospermum australe & Black bean & $\ldots$ & $\ldots$ & Northeast Australia, New Caledonia & $\mathrm{Y}$ & $\mathrm{Y}$ & $\ldots$ & $\mathrm{Y}$ \\
\hline \multirow[t]{2}{*}{ *Cercidium floridum } & Blue palo verde & $\ldots$ & $\ldots$ & Southwest United States, & & & & \\
\hline & & & & Western Mexico & $\mathrm{Y}$ & $\mathrm{Y}$ & $\ldots$ & $\ldots$ \\
\hline${ }^{*}$ C. $\times$ sonorae & Brea & $\ldots$ & $\ldots$ & Northwest Mexico & $\mathrm{Y}$ & $\mathrm{Y}$ & $\ldots$ & $\ldots$ \\
\hline Cladrastris sinensis & Chinese yellow wood & $\ldots$ & $\ldots$ & China & $\ldots$ & $\mathrm{Y}$ & $\ldots$ & $\mathrm{Y}$ \\
\hline Erythrina folkersii & Coral tree & $\ldots$ & $\ldots$ & Central America & $\mathrm{Y}$ & $\ldots$ & $\mathrm{Y}$ & $\ldots$ \\
\hline E. coralloides & Flame coral tree & $\ldots$ & $\ldots$ & Arizona and Mexico & $\ldots$ & $\mathrm{Y}$ & $\ldots$ & $\ldots$ \\
\hline *Erythrina corallodendron & Coral tree & $\ldots$ & $\ldots$ & West Indies & $\mathrm{Y}$ & $\mathrm{Y}$ & $\ldots$ & $\ldots$ \\
\hline E. crista-galli & Ceibo & $\ldots$ & $\ldots$ & South America & $\mathrm{Y}$ & $\mathrm{Y}$ & $\ldots$ & $\mathrm{Y}$ \\
\hline E. humeana & Dwarf coral tree & $\ldots$ & $\ldots$ & South Africa, Mozambique & $\mathrm{Y}$ & $\mathrm{Y}$ & $\mathrm{Y}$ & $\ldots$ \\
\hline E. lysistemon & Common coral tree & $\ldots$ & $\ldots$ & South Africa & $\mathrm{Y}$ & $\mathrm{Y}$ & $\mathrm{Y}$ & $\ldots$ \\
\hline E. $\times$ sykesii & Coral tree & $\ldots$ & $\ldots$ & Horticultural origin & $\mathrm{Y}$ & $\mathrm{Y}$ & $\ldots$ & $\ldots$ \\
\hline Inga feuilleii & Pacay & $\ldots$ & $\ldots$ & South America & $\mathrm{Y}$ & $\mathrm{Y}$ & $\ldots$ & $\ldots$ \\
\hline Lysiphylllum carronii & Queensland ebony & $\ldots$ & $\ldots$ & Australia & $\mathrm{Y}$ & $\ldots$ & $\ldots$ & $\mathrm{Y}$ \\
\hline *Parkinsonia aculeata & Palo verde & $\ldots$ & $\ldots$ & Tropical America & $\mathrm{Y}$ & $\mathrm{Y}$ & $\ldots$ & $\ldots$ \\
\hline Pithecellobium sp. & $\ldots$ & $\ldots$ & $\ldots$ & $\ldots$ & $\ldots$ & $\ldots$ & $\ldots$ & $\mathrm{Y}$ \\
\hline Schotia brachypetala & Huilboerboon & $\ldots$ & $\ldots$ & Southern Africa & $\mathrm{Y}$ & $\mathrm{Y}$ & $\ldots$ & $\mathrm{Y}$ \\
\hline Senna racemosa & Limestone senna & $\ldots$ & $\ldots$ & Central and South America & $\ldots$ & $\mathrm{Y}$ & $\ldots$ & $\ldots$ \\
\hline Senna racemosa var. liebmanni & $\ldots$ & $\ldots$ & $\ldots$ & Central and South America & $\mathrm{Y}$ & $\mathrm{Y}$ & $\ldots$ & $\ldots$ \\
\hline S. spectabilis var. spectabilis & $\ldots$ & $\ldots$ & $\ldots$ & $\ldots$ & $\ldots$ & $\mathrm{Y}$ & $\mathrm{Y}$ & $\mathrm{Y}$ \\
\hline \multicolumn{9}{|l|}{ Styphnolobium japonicum } \\
\hline (syn. Sophora japonica) & Pagoda tree & $\ldots$ & $\ldots$ & East Asia & $\ldots$ & $\ldots$ & $\ldots$ & $\mathrm{Y}$ \\
\hline Tipuana tipu & Tipu tree & 1 & $\ldots$ & Brazil, Argentina, Bolivia & $\ldots$ & $\mathrm{Y}$ & $\ldots$ & $\ldots$ \\
\hline Wisteria sinensis & Chinese wisteria & $\ldots$ & $\ldots$ & China & $\mathrm{Y}$ & $\ldots$ & $\ldots$ & $\ldots$ \\
\hline W. floribunda 'Macrobotyrus' & Japanese wisteria & $\ldots$ & $\ldots$ & Japan & $\mathrm{Y}$ & $\mathrm{Y}$ & $\ldots$ & $\ldots$ \\
\hline Zenia insignis & $\ldots$ & $\ldots$ & $\ldots$ & South China and Vietnam & $\ldots$ & $\mathrm{Y}$ & $\ldots$ & $\ldots$ \\
\hline \multicolumn{9}{|l|}{ Fagaceae } \\
\hline Fagus sylvatica & European beech & $\ldots$ & $\ldots$ & Central Europe to Caucasus & $\mathrm{Y}$ & $\mathrm{Y}$ & $\ldots$ & $\ldots$ \\
\hline F. sylvatica 'Riversii' & Riversii European Beech & $\ldots$ & $\ldots$ & Horticultural origin & $\ldots$ & $\ldots$ & $\mathrm{Y}$ & $\ldots$ \\
\hline Quercus acutissima & Sawtooth oak & $\ldots$ & $\ldots$ & China, Korea and Japan & $\ldots$ & $\ldots$ & $\ldots$ & $\ldots$ \\
\hline${ }^{*} Q$. agrifolia & Coast live oak & 13 & $\ldots$ & California, Baja California & $\mathrm{Y}$ & $\mathrm{Y}$ & $\ldots$ & $\ldots$ \\
\hline Q. alba & White oak & $\ldots$ & $\ldots$ & Eastern North America & $\ldots$ & $\ldots$ & $\ldots$ & $\ldots$ \\
\hline Q. chrysolepis & Canyon live oak & $\ldots$ & $\ldots$ & Western North America & $\mathrm{Y}$ & $\mathrm{Y}$ & $\ldots$ & $\ldots$ \\
\hline Q. engelmannii & Engelmann Oak & $\ldots$ & $\ldots$ & Southwest California & $\ldots$ & $\mathrm{Y}$ & $\ldots$ & $\ldots$ \\
\hline Q. ilex & Holly oak & 306 & $\ldots$ & Mediterranean & $\mathrm{Y}$ & $\mathrm{Y}$ & $\ldots$ & $\ldots$ \\
\hline Q. lobata & Valley oak & $\ldots$ & $\ldots$ & California & $\mathrm{Y}$ & $\mathrm{Y}$ & $\ldots$ & $\ldots$ \\
\hline Q. macrocarpa & Bur oak & $\ldots$ & $\ldots$ & Central and Eastern North America & $\mathrm{Y}$ & $\mathrm{Y}$ & $\ldots$ & $\ldots$ \\
\hline Q. mexicana & Cozahuatl & $\ldots$ & $\ldots$ & Mexico & $\mathrm{Y}$ & $\mathrm{Y}$ & $\ldots$ & $\mathrm{Y}$ \\
\hline${ }^{*} Q$. robur & English oak & $\ldots$ & $\ldots$ & Europe, Anatolia, and North Africa & $\mathrm{Y}$ & $\mathrm{Y}$ & $\ldots$ & $\ldots$ \\
\hline Q. rubra & Northern red oak & 7 & $\ldots$ & Eastern North America & $\ldots$ & $\mathrm{Y}$ & $\mathrm{Y}$ & $\mathrm{Y}$ \\
\hline \multirow{2}{*}{ Q. suber } & Cork oak & $\ldots$ & $\cdots$ & Southwest Europe and & & & & \\
\hline & & & & Northwest Africa & $\mathrm{Y}$ & $\mathrm{Y}$ & $\ldots$ & $\cdots$ \\
\hline Q. virginiana & Southern live oak & $\ldots$ & $\ldots$ & Southeastern United States & $\mathrm{Y}$ & $\mathrm{Y}$ & $\ldots$ & $\ldots$ \\
\hline \multicolumn{9}{|c|}{ Hamamelidaceae s.l. (Altingiaceae s.s.) } \\
\hline Liquidambar formosana & Chinese sweet gum & 12 & $\ldots$ & South China, Taiwan & $\mathrm{Y}$ & $\mathrm{Y}$ & $\ldots$ & $\ldots$ \\
\hline *L. styraciflua & American sweetgum & 4,916 & $\ldots$ & Eastern North America & $\mathrm{Y}$ & $\mathrm{Y}$ & $\ldots$ & $\ldots$ \\
\hline \multicolumn{9}{|c|}{ Hydrophyllaceae s.s. (Boraginaceae s.l.) } \\
\hline Wigandia urens & $\ldots$ & $\ldots$ & $\ldots$ & Peru & $\ldots$ & $\mathrm{Y}$ & $\ldots$ & $\ldots$ \\
\hline Juglandaceae & & & & & & & & \\
\hline Carya illinoinensis & Hickory & 36 & $\ldots$ & South-Central North America & $\mathrm{Y}$ & $\mathrm{Y}$ & $\ldots$ & $\mathrm{Y}$ \\
\hline Jugans mandshurica & & & & & & & & \\
\hline (syn. Juglans formosana) & Hayata & $\ldots$ & $\ldots$ & China, Taiwan & $\ldots$ & $\ldots$ & $\mathrm{Y}$ & $\ldots$ \\
\hline J. nigra & Eastern black walnut & 5 & $\mathrm{Y}$ & Eastern North America & $\ldots$ & $\mathrm{Y}$ & $\ldots$ & $\ldots$ \\
\hline Pterocarya sp. & Wingnut tree & $\ldots$ & $\ldots$ & Asia & $\ldots$ & $\mathrm{Y}$ & $\ldots$ & $\mathrm{Y}$ \\
\hline & & & & & continu & edo & next & age) \\
\hline
\end{tabular}


Table 1. (continued from preceding page)

\begin{tabular}{|c|c|c|c|c|c|c|c|c|}
\hline Family, species ${ }^{\mathbf{b}}$ & Common name & $N^{\mathrm{c}}$ & Crop $^{d}$ & Origin & FD $^{\mathbf{e}}$ & $\mathbf{S}$ & $\mathbf{P}$ & G \\
\hline \multicolumn{9}{|l|}{ Lauraceae } \\
\hline Cinпатотит glanduliferum & False camphor tree & & $\ldots$ & China & & $\ldots$ & $\ldots$ & $\ldots$ \\
\hline C. camphora & Camphor tree & 690 & $\ldots$ & Japan to Tropical Asia & $\mathrm{Y}$ & $\mathrm{Y}$ & $\mathrm{Y}$ & $\ldots$ \\
\hline Nothaphoebe cavalieri & $\ldots$ & $\ldots$ & $\ldots$ & South China & $\ldots$ & $\mathrm{Y}$ & $\ldots$ & $\ldots$ \\
\hline *Persea americana & Avocado & 70 & $\mathrm{Y}$ & Mexico, Guatamala, West Indies & $\mathrm{Y}$ & $\mathrm{Y}$ & $\mathrm{Y}$ & $\ldots$ \\
\hline \multicolumn{9}{|l|}{ Machilus thunbergii } \\
\hline (syn. Persea thunbergii) & Asian avocado & $\ldots$ & $\ldots$ & East Asia & Y & $\mathrm{Y}$ & $\ldots$ & $\ldots$ \\
\hline Umbellularia californica & California bay laurel & $\ldots$ & $\ldots$ & Western North America & $\mathrm{Y}$ & $\mathrm{Y}$ & $\ldots$ & $\ldots$ \\
\hline \multicolumn{9}{|l|}{ Magnoliaceae } \\
\hline Magnolia 'Columbus' & Columbus magnolia & $\ldots$ & $\ldots$ & Horticultural origin & $\ldots$ & Y & $\ldots$ & $\ldots$ \\
\hline M. delavayi & Chinese evergreen & & & & & & & \\
\hline & magnolia & $\ldots$ & $\ldots$ & Southwest China & $\ldots$ & $\mathrm{Y}$ & $\ldots$ & $\ldots$ \\
\hline M. doltsopa & Sweet michelia & $\ldots$ & $\ldots$ & Southwest China, Himalayas & $\ldots$ & $\mathrm{Y}$ & $\ldots$ & $\ldots$ \\
\hline M. grandiflora & Southern magnolia & 2,174 & $\ldots$ & Southeast United States & $\mathrm{Y}$ & $\mathrm{Y}$ & $\ldots$ & $\ldots$ \\
\hline M. guatemalensis & Mamey & $\ldots$ & $\ldots$ & Central America & $\ldots$ & $\mathrm{Y}$ & $\ldots$ & $\mathrm{Y}$ \\
\hline M. $\times$ soulangeana & Saucer magnolia & 8 & $\ldots$ & Horticultural origin & $\ldots$ & $\mathrm{Y}$ & $\ldots$ & $\mathrm{Y}$ \\
\hline M. $\times$ veitchii & Veitch's magnolia & $\ldots$ & $\ldots$ & Horticultural origin & $\mathrm{Y}$ & $\mathrm{Y}$ & $\ldots$ & $\ldots$ \\
\hline \multicolumn{9}{|l|}{ Malvaceae } \\
\hline Dombeya саситіпит & Strawberry tree & $\ldots$ & $\ldots$ & Madagascar & $\mathrm{Y}$ & $\ldots$ & $\mathrm{Y}$ & $\ldots$ \\
\hline Brachychiton acerifolius & Illawarra flame tree & 46 & $\ldots$ & Northeast Australia & $\mathrm{Y}$ & $\mathrm{Y}$ & $\ldots$ & $\mathrm{Y}$ \\
\hline B. australis & Kurrajong & $\ldots$ & $\ldots$ & Northern Australia & $\mathrm{Y}$ & $\mathrm{Y}$ & $\ldots$ & $\ldots$ \\
\hline B. discolor & lacebark tree & $\ldots$ & $\ldots$ & Northeast Australia & $\mathrm{Y}$ & $\ldots$ & $\ldots$ & $\ldots$ \\
\hline B. rupestris & Queensland bottle tree & $\ldots$ & $\ldots$ & Northeast Australia & $\mathrm{Y}$ & $\ldots$ & $\ldots$ & $\mathrm{Y}$ \\
\hline Chiranthodendron pentadactylon & Devil's hand tree & $\ldots$ & $\ldots$ & Mexico and Central America & $\ldots$ & $\mathrm{Y}$ & $\ldots$ & $\mathrm{Y}$ \\
\hline Firmiana simplex & Chinese parasol tree & $\ldots$ & $\ldots$ & East Asia & $\mathrm{Y}$ & $\ldots$ & $\ldots$ & $\ldots$ \\
\hline \multicolumn{9}{|l|}{ Meliaceae } \\
\hline Melia azedarach & Cape lilac & 11 & $\ldots$ & India & $\ldots$ & $\mathrm{Y}$ & $\ldots$ & $\ldots$ \\
\hline \multicolumn{9}{|l|}{ Swietenia chickrassa } \\
\hline (syn. Chukrasia tabularis) & $\ldots$ & $\ldots$ & $\ldots$ & India and China & $\ldots$ & $\ldots$ & $\ldots$ & $\mathrm{Y}$ \\
\hline \multicolumn{9}{|l|}{ Melianthaceae } \\
\hline Melianthus major & Giant honey flower & $\ldots$ & $\ldots$ & South Africa & $\mathrm{Y}$ & $\ldots$ & $\ldots$ & $\ldots$ \\
\hline Menispermaceae & & & & & & & & \\
\hline Cocculus laurifolius & Laurel-leaved snail tree & $\ldots$ & $\ldots$ & Himalayas to Japan & $\mathrm{Y}$ & $\mathrm{Y}$ & $\ldots$ & $\ldots$ \\
\hline C. orbiculatus (syn. C. trilobus) & Korean moonseed & $\ldots$ & $\ldots$ & East and Southeast Asia & $\mathrm{Y}$ & $\mathrm{Y}$ & $\ldots$ & $\mathrm{Y}$ \\
\hline Monimiaceae & & & & & & & & \\
\hline Peumus boldus & Boldo & $\ldots$ & $\ldots$ & Chile & $\ldots$ & $\mathrm{Y}$ & $\ldots$ & $\ldots$ \\
\hline Moraceae & & & & & & & & \\
\hline Broussonetia papyrifera & Paper mulberry & $\ldots$ & $\ldots$ & Eastern Asia & $\ldots$ & $\mathrm{Y}$ & $\ldots$ & $\ldots$ \\
\hline Ficus benjamina & Weeping fig & 1,182 & $\ldots$ & Southeast Asia and Australia & $\ldots$ & $\mathrm{Y}$ & $\ldots$ & $\ldots$ \\
\hline F. macrophylla & Moreton Bay fig & 2 & $\ldots$ & Northeast Australia & $\mathrm{Y}$ & $\mathrm{Y}$ & $\ldots$ & $\mathrm{Y}$ \\
\hline F. maxima & Fig & $\ldots$ & $\ldots$ & Tropical America & $\ldots$ & $\ldots$ & $\ldots$ & $\ldots$ \\
\hline F. platypoda & Desert rock fig & $\ldots$ & $\ldots$ & Australia and Indonesia & $\mathrm{Y}$ & $\mathrm{Y}$ & $\ldots$ & $\mathrm{Y}$ \\
\hline Morus alba & White mulberry & 43 & $\mathrm{Y}$ & China & $\mathrm{Y}$ & $\mathrm{Y}$ & $\ldots$ & $\ldots$ \\
\hline Myrtaceae & & & & & & & & \\
\hline Callistemon salignus & Willow bottlebrush & $\ldots$ & $\ldots$ & Australia & $\ldots$ & $\ldots$ & $\ldots$ & $\ldots$ \\
\hline C. viminalis & Weeping bottlebrush & 91 & $\ldots$ & Australia & $\ldots$ & $\mathrm{Y}$ & $\ldots$ & $\ldots$ \\
\hline Eucalyptus camaldulensis & River red gum & 2 & $\ldots$ & Australia & $\ldots$ & $\ldots$ & $\ldots$ & $\ldots$ \\
\hline E. cinerea & Argyle apple & $\ldots$ & $\ldots$ & Southeast Australia & $\ldots$ & $\mathrm{Y}$ & $\ldots$ & $\ldots$ \\
\hline E. ficifolia & Red flowering gum & 33 & $\ldots$ & Western Australia & $\ldots$ & $\mathrm{Y}$ & $\ldots$ & $\mathrm{Y}$ \\
\hline E. froggattii & $\ldots$ & $\ldots$ & $\ldots$ & Southeast Australia & $\ldots$ & Y & $\ldots$ & $\mathrm{Y}$ \\
\hline E. kitsoniana & Gippsland mallee & $\ldots$ & $\ldots$ & Southeast Australia & $\ldots$ & $\mathrm{Y}$ & $\ldots$ & $\ldots$ \\
\hline E. perriniana & Spinning gum & $\ldots$ & $\ldots$ & Southeast Australia & $\ldots$ & $\mathrm{Y}$ & $\ldots$ & $\mathrm{Y}$ \\
\hline E. polyanthemos & Red box & 6 & $\ldots$ & Southeast Australia & $\mathrm{Y}$ & Y & $\ldots$ & $\ldots$ \\
\hline E. torquata & Coral gum & $\ldots$ & $\ldots$ & Western Australia & $\mathrm{Y}$ & $\mathrm{Y}$ & $\ldots$ & $\ldots$ \\
\hline Nyssaceae s.s. (Cornaceae s.1.) & & & & & & & & \\
\hline Camptotheca acuminata & Happy tree & $\ldots$ & $\ldots$ & China and Tibet & $\mathrm{Y}$ & $\mathrm{Y}$ & $\ldots$ & $\ldots$ \\
\hline Oleaceae & & & & & & & & \\
\hline Chionanthus retusus & Chinese fringetree & $\ldots$ & $\ldots$ & East Asia & $\mathrm{Y}$ & Y & $\ldots$ & $\ldots$ \\
\hline Fraxinus sp. & Ash & $\ldots$ & $\ldots$ & & $\ldots$ & $\mathrm{Y}$ & $\ldots$ & $\ldots$ \\
\hline F. uhdei & Shamel ash & 48 & $\ldots$ & Central America & $\mathrm{Y}$ & $\mathrm{Y}$ & $\ldots$ & $\mathrm{Y}$ \\
\hline F. velutina & Velvet ash & 57 & $\ldots$ & Southwestern North America & $\ldots$ & $\mathrm{Y}$ & $\ldots$ & \\
\hline Olea europaea & Olive & 29 & $\mathrm{Y}$ & Mediterranean, Asia and Africa & $\mathrm{Y}$ & $\mathrm{Y}$ & $\ldots$ & $\mathrm{Y}$ \\
\hline Osmanthus fragrans & Sweet osmanthus & $\ldots$ & $\ldots$ & Himalayas to Japan & $\ldots$ & $\mathrm{Y}$ & $\mathrm{Y}$ & $\ldots$ \\
\hline Onagraceae & & & & & & & & \\
\hline Hauya microcerata & $\ldots$ & $\ldots$ & $\ldots$ & Central America & $\ldots$ & $\ldots$ & $\ldots$ & $\ldots$ \\
\hline Pinaceae & & & & & & & & \\
\hline Cedrus atlantica & Atlas cedar & 1 & $\ldots$ & Algeria and Morocco & $\ldots$ & $\mathrm{Y}$ & $\ldots$ & $\mathrm{Y}$ \\
\hline Keteleeria delavayi (syn. K. evelyniana) & $\ldots$ & $\ldots$ & $\ldots$ & South China, Laos and Vietnam & $\ldots$ & $\mathrm{Y}$ & $\ldots$ & $\ldots$ \\
\hline Pinus densiflora & Japanese red pine & $\ldots$ & $\ldots$ & Japan, Korea, China & $\ldots$ & $\mathrm{Y}$ & $\ldots$ & $\mathrm{Y}$ \\
\hline P. douglasiana & $\ldots$ & $\ldots$ & $\ldots$ & Western Mexico & $\ldots$ & $\ldots$ & $\ldots$ & $\mathrm{Y}$ \\
\hline Pittosporaceae & & & & & & & & \\
\hline Hymenosporum flavum & Native frangipani & 20 & $\ldots$ & Australia and New Guinea & $\mathrm{Y}$ & $\ldots$ & $\ldots$ & $\mathrm{Y}$ \\
\hline Pittosporum undulatum & Victorian box & $\cdots$ & $\cdots$ & Eastern Australia & Y & $\mathrm{Y}$ & $\ldots$ & $\mathrm{Y}$ \\
\hline & & & & & (continu & d on & ext & age) \\
\hline
\end{tabular}


HCO2198 (5'-TAAACTTCAGGGTGACCAAAAAATCA-3') (8). PCR and sequencing was performed as described by RugmanJones et al. (23). Sequences were aligned manually using BioEdit, version 7.0.9.0 (9). Primers were removed and each sequence was translated (http://www.ebi.ac.uk/Tools/emboss/transeq/index.html) to confirm the absence of nuclear pseudogenes (26). All specimens were confirmed as PSHB (and not TSHB) by comparison with GenBank accession numbers JX912723-JX912725.

Table 1. (continued from preceding page)

\begin{tabular}{|c|c|c|c|c|c|c|c|c|}
\hline Family, species ${ }^{b}$ & Common name & $N^{\mathrm{c}}$ & Crop $^{d}$ & Origin & $\mathbf{F D}^{\mathbf{e}}$ & $\mathbf{S}$ & $\mathbf{P}$ & $\mathbf{G}$ \\
\hline \multicolumn{9}{|l|}{ Platanaceae } \\
\hline Platanus mexicana & Mexican sycamore & $\ldots$ & $\ldots$ & Northeast Mexico & $\mathrm{Y}$ & $\mathrm{Y}$ & $\ldots$ & $\ldots$ \\
\hline P. occidentalis & American sycamore & 19 & $\ldots$ & Eastern North America & $\mathrm{Y}$ & $\mathrm{Y}$ & $\ldots$ & $\ldots$ \\
\hline *P. racemosa & California sycamore & 68 & $\ldots$ & California, Baja California & $\mathrm{Y}$ & $\mathrm{Y}$ & $\ldots$ & $\ldots$ \\
\hline P. wrightii & Arizona sycamore & $\ldots$ & $\cdots$ & $\begin{array}{l}\text { Southwest United States, } \\
\text { Northwest Mexico }\end{array}$ & $\mathrm{Y}$ & $\ldots$ & $\ldots$ & $\ldots$ \\
\hline \multicolumn{9}{|l|}{ Poaceae } \\
\hline \multicolumn{8}{|l|}{ Podocarpaceae } & $\cdots$ \\
\hline $\begin{array}{l}\text { Afrocarpus gracilior (syn. Podocarpus } \\
\text { gracilior, Decussocarpus gracilior) }\end{array}$ & Fern pine & $\cdots$ & $\cdots$ & East Africa & $\cdots$ & $\cdots$ & $\cdots$ & $\cdots$ \\
\hline \multicolumn{9}{|l|}{ Proteaceae } \\
\hline Banksia saxicola & Grampians banksia & $\ldots$ & $\ldots$ & Southeast Australia & $\mathrm{Y}$ & $\mathrm{Y}$ & $\ldots$ & $\ldots$ \\
\hline Macadamia integrifolia & Macadamia nut & $\ldots$ & $\mathrm{Y}$ & Eastern Australia & $\mathrm{Y}$ & $\mathrm{Y}$ & $\ldots$ & $\ldots$ \\
\hline \multicolumn{9}{|l|}{ Rhamnaceae } \\
\hline Rhamnus californica & Coffee berry & $\ldots$ & $\ldots$ & Western North America & $\ldots$ & $\ldots$ & $\ldots$ & $\mathrm{Y}$ \\
\hline Ziziphus jujuba & Chinese date & $\ldots$ & $\ldots$ & Temperate Asia & $\mathrm{Y}$ & $\mathrm{Y}$ & $\ldots$ & $\ldots$ \\
\hline \multicolumn{9}{|l|}{ Rosaceae } \\
\hline Eriobotrya japonica & Loquat & 4 & $\mathrm{Y}$ & China, Japan & $\mathrm{Y}$ & $\ldots$ & $\ldots$ & $\ldots$ \\
\hline Malus floribunda & Japanese crabapple & 2 & $\mathrm{Y}$ & East Asia & $\ldots$ & $\mathrm{Y}$ & $\ldots$ & $\mathrm{Y}$ \\
\hline Prunus cerasoides & Wild Himalayan cherry & $\ldots$ & $\ldots$ & South Asia & $\ldots$ & $\ldots$ & $\ldots$ & $\mathrm{Y}$ \\
\hline P. caroliniana & Wild mock orange & $\ldots$ & $\ldots$ & Southeastern United States & $\ldots$ & $\mathrm{Y}$ & $\ldots$ & $\mathrm{Y}$ \\
\hline P. mите & Chinese plum & $\ldots$ & $\ldots$ & Southern Japan & Y & $\ldots$ & $\ldots$ & $\mathrm{Y}$ \\
\hline P. persica & Peach & 4 & $\mathrm{Y}$ & China & $\mathrm{Y}$ & $\mathrm{Y}$ & $\ldots$ & $\mathrm{Y}$ \\
\hline P. serrulata & Japanese cherry & $\ldots$ & $\ldots$ & Japan, Korea and China & $\mathrm{Y}$ & $\ldots$ & $\ldots$ & $\mathrm{Y}$ \\
\hline \multicolumn{9}{|l|}{ Chaenomeles sinensis } \\
\hline (syn. Cydonia sinensis) & Chinese quince & $\ldots$ & $\ldots$ & China & $\ldots$ & $\ldots$ & $\ldots$ & $\ldots$ \\
\hline Pyrus kawakamii & Evergreen pear & 156 & $\ldots$ & China, Taiwan & $\mathrm{Y}$ & $\mathrm{Y}$ & $\ldots$ & $\ldots$ \\
\hline \multicolumn{9}{|l|}{ Rutaceae } \\
\hline Citrus sinensis & Orange & 6 & $\mathrm{Y}$ & Southeast Asia & $\mathrm{Y}$ & $\mathrm{Y}$ & $\ldots$ & $\mathrm{Y}$ \\
\hline \multicolumn{9}{|l|}{ Salicaceae } \\
\hline Salix babylonica & Babylon willow & 1 & $\ldots$ & Asia & $\ldots$ & $\mathrm{Y}$ & $\mathrm{Y}$ & $\mathrm{Y}$ \\
\hline Salix sp. & $\ldots$ & $\ldots$ & $\ldots$ & $\ldots$ & $\mathrm{Y}$ & $\mathrm{Y}$ & $\mathrm{Y}$ & $\mathrm{Y}$ \\
\hline Xylosma congestum & Dense logwood & $\ldots$ & $\ldots$ & China & $\mathrm{Y}$ & $\mathrm{Y}$ & $\ldots$ & $\ldots$ \\
\hline \multicolumn{9}{|l|}{ Sapindaceae } \\
\hline Alectryon excelsus & Tītoki & $\ldots$ & $\ldots$ & New Zealand & $\mathrm{Y}$ & $\mathrm{Y}$ & $\ldots$ & $\mathrm{Y}$ \\
\hline Harpullia arborea & Tulip-wood tree & $\ldots$ & $\ldots$ & Tropical Southeast Asia & $\mathrm{Y}$ & $\mathrm{Y}$ & $\mathrm{Y}$ & \\
\hline H. pendula & Tulipwood & $\ldots$ & $\ldots$ & $\begin{array}{l}\text { Northeast Australia, } \\
\text { Tropical Southeast Asia }\end{array}$ & $\cdots$ & $\mathrm{Y}$ & $\mathrm{Y}$ & $\mathrm{Y}$ \\
\hline Koelreuteria bipinnata & Chinese flame tree & 30 & $\ldots$ & Southwest China & $\ldots$ & $\mathrm{Y}$ & $\ldots$ & $\mathrm{Y}$ \\
\hline K. elegans & Chinese rain tree & $\ldots$ & $\ldots$ & Taiwan and Southern China & $\ldots$ & $\mathrm{Y}$ & $\ldots$ & $\mathrm{Y}$ \\
\hline \multicolumn{9}{|l|}{ K. elegans subsp. formosana } \\
\hline (syn. K. henryi) & $\ldots$ & $\ldots$ & $\ldots$ & Taiwan & $\ldots$ & $\mathrm{Y}$ & $\ldots$ & $\mathrm{Y}$ \\
\hline Ungnadia speciosa & Mexican buckeye & $\ldots$ & $\ldots$ & South United States, North Mexico & $\mathrm{Y}$ & $\mathrm{Y}$ & $\ldots$ & $\ldots$ \\
\hline Simaroubaceae & & & & & & & & \\
\hline Ailanthus altissima & Tree of heaven & 2 & $\ldots$ & China and Taiwan & $\ldots$ & $\ldots$ & $\ldots$ & $\mathrm{Y}$ \\
\hline Taxodiaceae s.s. (Cupressaceae s.1.) & & & & & & & & \\
\hline Metasequoia glyptostroboides** & Dawn redwood & $\ldots$ & $\ldots$ & Western China & $\mathrm{Y}$ & $\ldots$ & $\ldots$ & $\mathrm{Y}$ \\
\hline Theaceae & & & & & & & & \\
\hline Camellia japonica & Japanese camellia & $\ldots$ & $\ldots$ & Japan, Korea and China & $\ldots$ & $\ldots$ & $\mathrm{Y}$ & $\ldots$ \\
\hline Camellia 'Apple Blossom’ & Apple Blossom camellia & $\ldots$ & $\ldots$ & Horticultural origin & $\ldots$ & $\mathrm{Y}$ & $\ldots$ & $\ldots$ \\
\hline Camellia 'Pink Sparkle' & Pink Sparkle camellia & $\ldots$ & $\ldots$ & Horticultural origin & $\ldots$ & $\mathrm{Y}$ & $\ldots$ & $\ldots$ \\
\hline C. reticulata & Camellia & $\ldots$ & $\ldots$ & Southwest China & $\mathrm{Y}$ & $\mathrm{Y}$ & $\ldots$ & $\ldots$ \\
\hline${ }^{*}$ C. semiserrata & Camellia & $\ldots$ & $\ldots$ & $\ldots$ & $\mathrm{Y}$ & $\ldots$ & $\ldots$ & $\ldots$ \\
\hline Cleyera japonica & Sakaki & $\ldots$ & $\ldots$ & East Asia & $\mathrm{Y}$ & $\mathrm{Y}$ & $\ldots$ & $\ldots$ \\
\hline Tiliaceae s.s. (Malvaceae s.1.) & & & & & & & & \\
\hline Heliocarpus donnellsmithii & $\ldots$ & $\ldots$ & $\ldots$ & Mexico and Central America & $\mathrm{Y}$ & $\mathrm{Y}$ & $\ldots$ & $\ldots$ \\
\hline Luehea divaricata & Açoita-cavalo & $\ldots$ & $\ldots$ & Brazil & $\mathrm{Y}$ & $\mathrm{Y}$ & $\mathrm{Y}$ & $\mathrm{Y}$ \\
\hline Tilia americana & Basswood & 1 & $\ldots$ & Central and Eastern North America & $\mathrm{Y}$ & $\mathrm{Y}$ & $\mathrm{Y}$ & $\ldots$ \\
\hline Ulmaceace & & & & & & & & \\
\hline Ulmus alata & Winged elm & $\ldots$ & $\ldots$ & Central and Eastern United States & $\ldots$ & $\mathrm{Y}$ & $\ldots$ & $\ldots$ \\
\hline U. americana & American elm & 1 & $\ldots$ & Eastern North America & $\mathrm{Y}$ & $\ldots$ & $\ldots$ & $\ldots$ \\
\hline U. parvifolia & Chinese elm & 183 & $\ldots$ & East Asia & $\mathrm{Y}$ & $\mathrm{Y}$ & $\ldots$ & $\ldots$ \\
\hline Zelkova serrata & Ju shu & 1 & $\ldots$ & East Asia & Y & $\ldots$ & $\ldots$ & $\ldots$ \\
\hline Urticaceae & & & & & & & & \\
\hline Pipturus argenteus & $\ldots$ & $\ldots$ & $\ldots$ & Australia, Tropical Asia & $\mathrm{Y}$ & $\mathrm{Y}$ & $\mathrm{Y}$ & $\ldots$ \\
\hline Verbenaceae & & & & & & & & \\
\hline Aloysia virgate & Sweet almond bush & $\ldots$ & $\ldots$ & South America & $\ldots$ & $\ldots$ & $\ldots$ & $\ldots$ \\
\hline Vitaceae & & & & & & & & \\
\hline Vitis vinifera & Grapevine & $\ldots$ & $\mathrm{Y}$ & Mediterranean, Europe & $\mathrm{Y}$ & $\ldots$ & $\ldots$ & $\mathrm{Y}$ \\
\hline
\end{tabular}


Table 2. Apparent nonhosts of polyphagous shot hole borer

\begin{tabular}{|c|c|c|c|}
\hline Family, species & Common name & Origin & $N$ planted $^{\mathrm{a}}$ \\
\hline \multicolumn{4}{|l|}{ Aceraceae s.s. (Sapindaceae s.1.) } \\
\hline Acer rubrum & Red maple & Eastern North America & 1 \\
\hline \multicolumn{4}{|l|}{ Adoxaceae } \\
\hline Sambucus mexicana & Mexican elderberry & Western North America & $\ldots$ \\
\hline Sambucus nigra & Black elderberry & Europe, North Africa, Southwest Asia & $\ldots$ \\
\hline \multicolumn{4}{|l|}{ Agavaceae s.s. (Asparagaceae s.1.) } \\
\hline Beaucarnea recurvata (syn. Nolina recurvata) & $\ldots$ & Mexico & $\ldots$ \\
\hline \multicolumn{4}{|l|}{ Aloeaceae s.s. (Xanthorrhoeaceae s.1.) } \\
\hline Aloe bainesii & $\ldots$ & Southern Africa & $\ldots$ \\
\hline Aloe dichotoma & $\ldots$ & Southern Africa & $\ldots$ \\
\hline \multicolumn{4}{|l|}{ Anacardiaceae } \\
\hline Rhus lancea & African sumac & South Africa & 18 \\
\hline \multicolumn{4}{|l|}{ Apocynaceae } \\
\hline Acokanthera oppositifolia & $\ldots$ & Tropical and Southern Africa & $\ldots$ \\
\hline Nerium oleander & Oleander & Mediterranean, Middle and Central Asia & 68 \\
\hline \multicolumn{4}{|l|}{ Arecaceae } \\
\hline Archontophoenix cunninghamiana & King palm & Eastern Australia & 15 \\
\hline \multicolumn{4}{|l|}{ Syagrus romanzoffianum } \\
\hline (syn. Arecastrum romanzoffianum) & Queen palm & South America & 426 \\
\hline Chamaerops humilis & Mediterranean fan palm & Mediterranean & 2 \\
\hline Phoenix canariensis & Canary Island date palm & Canary Islands & 34 \\
\hline P. dactylifera & Date palm & Unknown origin & 46 \\
\hline$P$. reclinata & Senegal date palm & Tropical Africa & $\ldots$ \\
\hline Trachycarpus fortunei & Chinese windmill palm & Asia & 80 \\
\hline \multicolumn{4}{|l|}{ Asteraceae } \\
\hline Montanoa guatemalensis & $\ldots$ & Central America & $\ldots$ \\
\hline \multicolumn{4}{|l|}{ Betulaceae } \\
\hline Betula nigra 'Cully' & Heritage river birch & Horticultural origin & $\ldots$ \\
\hline Carpinus caroliniana & America hornbeam & Eastern North America, Mexico, Central America & $\ldots$ \\
\hline Ostrya virginiana & American hophornbeam & Eastern North America & $\ldots$ \\
\hline Bignoniaceae & & & \\
\hline Jacaranda mimosifolia & Jacaranda & South America & 753 \\
\hline Handroanthus chrysotrichus & & & \\
\hline (syn. Tabebuia chrysotricha) & Golden trumpet tree & Brazil & 1 \\
\hline Boraginaceae & & & \\
\hline Ehretia latifolia (syn. E. austin-smithii) & $\ldots$ & Mexico, Central America & $\ldots$ \\
\hline Burseraceae & & & \\
\hline Bursera odorata & $\ldots$ & Mexico & $\ldots$ \\
\hline Cactaceae & & & \\
\hline Pereskia grandiflora & $\ldots$ & Brazil & $\ldots$ \\
\hline Caprifoliaceae & & & \\
\hline Weigela coraeensis & Japanese weigela & Eastern Asia & $\ldots$ \\
\hline Celastraceae & & & \\
\hline Euonymus bungeanus & $\ldots$ & China, Korea & $\ldots$ \\
\hline Maytenus boaria & Mayten & Chile & 5 \\
\hline Cercidiphyllaceae & & & \\
\hline Cercidiphyllum japonicum & Katsura tree & China, Japan & $\ldots$ \\
\hline Clethraceae & & & \\
\hline Clethra macrophylla & Mexican clethra & Mexico & $\ldots$ \\
\hline Corynocarpaceae & & & \\
\hline Corynocarpus laevigatus & Karaka & New Zealand & $\ldots$ \\
\hline Cupressaceae & & & \\
\hline Calocedrus decurrens & California incense cedar & Western North America & 22 \\
\hline Juniperus bermudiana & Bermuda juniper & Bermuda & $\ldots$ \\
\hline Ericaceae & & & \\
\hline Erica $\times$ darleyensis & $\ldots$ & Horticultural origin & $\ldots$ \\
\hline Fabaceae & & & \\
\hline Ceratonia siliqua & Carob & Mediterranean, North Africa, Middle East and & \\
\hline & & Western Asia & 15 \\
\hline Millettia reticulata & Evergreen wisteria & South China, Taiwan & $\ldots$ \\
\hline Pithecellobium dulce & Huamuchil & Mexico, Central America & $\ldots$ \\
\hline Sophora secundiflora & Mountain laurel & Southwest United States, Mexico & $\ldots$ \\
\hline Fagaceae & & & \\
\hline Lithocarpus glaber & $\ldots$ & Japan, East China & $\ldots$ \\
\hline Quercus emoryi & Emory oak & Southwest United States, Mexico & $\ldots$ \\
\hline Q. variabilis & Chinese cork oak & China, Japan, Korea & $\ldots$ \\
\hline Fouquieriaceae & & & \\
\hline Fouquieria macdougalii & Mexican ocotillo & Northwest Mexico & $\ldots$ \\
\hline Garryaceae & & & \\
\hline Garrya wrightii & $\ldots$ & Southwest United States, Northwest Mexico & $\ldots$ \\
\hline Ginkgoaceae & & & \\
\hline Ginkgo biloba & Maidenhair tree & China & 231 \\
\hline & & (continued & on next page) \\
\hline
\end{tabular}

a Number of times planted along streets of City of Orange (4). 
Table 2. (continued from preceding page)

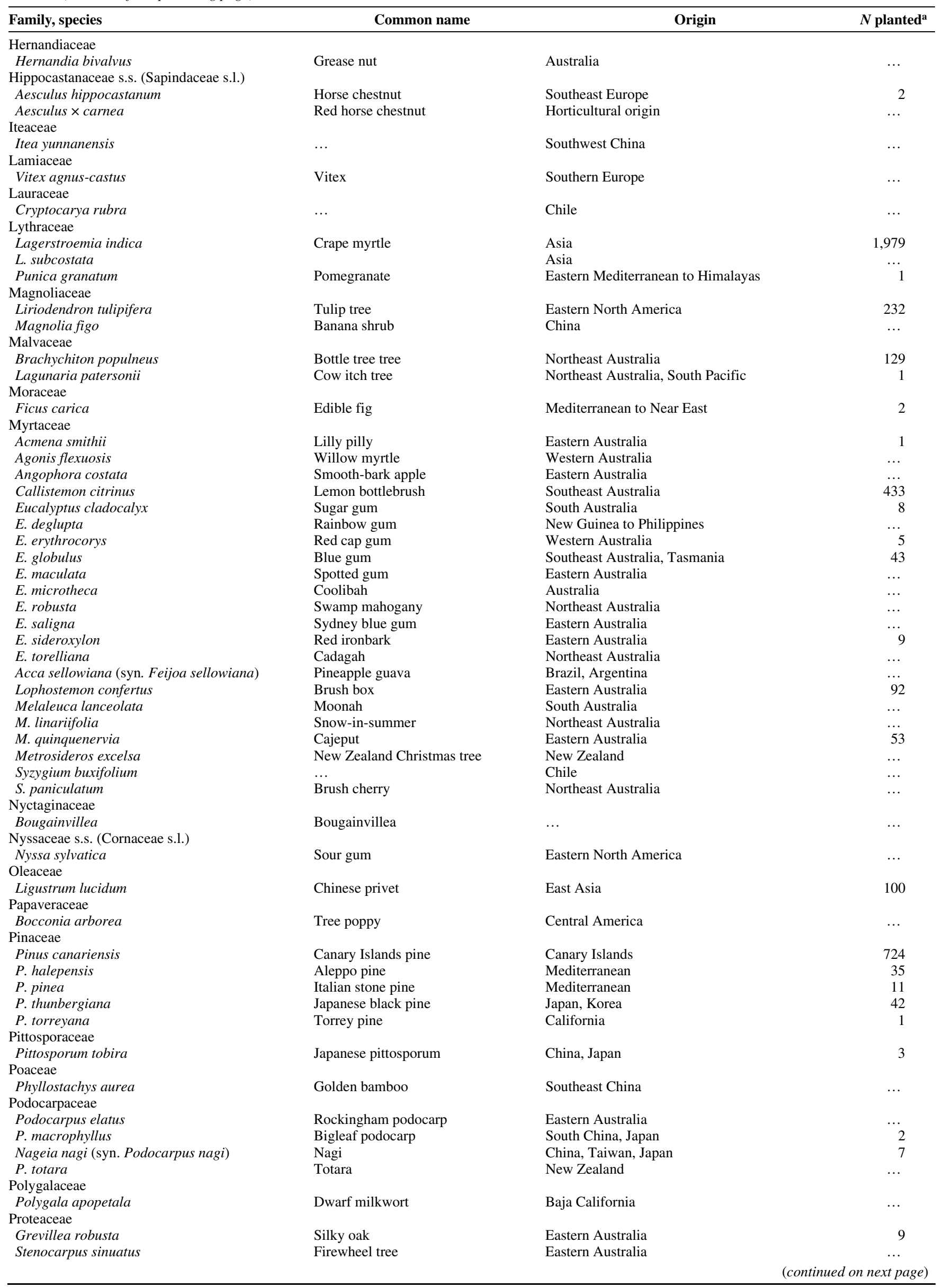


Table 2. (continued from preceding page)

\begin{tabular}{|c|c|c|c|}
\hline Family, species & Common name & Origin & $N$ planted \\
\hline \multicolumn{4}{|l|}{ Rhamnaceae } \\
\hline Hovenia dulcis & Raisin tree & East Asia & $\ldots$ \\
\hline Ziziphus spina-christi & $\ldots$ & East Africa, Middle and Near East & $\ldots$ \\
\hline \multicolumn{4}{|l|}{ Rosaceae } \\
\hline Crataegus laevigata & Midland hawthorn & Europe, North Africa, India & $\ldots$ \\
\hline Eriobotrya deflexa & Bronze loquat & China, Taiwan & $\ldots$ \\
\hline Malus syvestris & Apple & Central Europe & 1 \\
\hline Photinia $\times$ fraseri & Fraser's photinia & Horticultural origin & 14 \\
\hline Pyrus calleryana & Ornamental pear & East Asia & 2173 \\
\hline \multicolumn{4}{|l|}{ Rutaceae } \\
\hline Calodendrum capense & Cape chestnut & South Africa & 12 \\
\hline Casimiroa edulis & Cochitzapotl & Mexico and Central America & $\ldots$ \\
\hline Citrus limon & Lemon & Unknown origin & 5 \\
\hline C. sinensis 'Moro' & Moro blood orange & Horticultural origin & $\ldots$ \\
\hline Tetradium danieli (syn. Evodia danieli) & & China, Korea & $\ldots$ \\
\hline Geijera parviflora & Australian willow & Australia & 147 \\
\hline Melicope elleryana & Pink flowered soughwood & Australia & $\ldots$ \\
\hline$\times$ Citrofortunella & $\ldots$ & Horticultural origin & $\ldots$ \\
\hline \multicolumn{4}{|l|}{ Salicaceae } \\
\hline Dovyalis hebecarpa & Ketembilla & Sri Lanka, Southern India & $\ldots$ \\
\hline Olmediella betschleriana & Guatemalan holly & Mexico, Central America & $\ldots$ \\
\hline Oncoba spinosa & Snuff-box tree & Africa, Arabia & $\ldots$ \\
\hline \multicolumn{4}{|l|}{ Sciadopityaceae } \\
\hline Sciadopitys verticillata & Japanese umbrella-pine & Japan & $\ldots$ \\
\hline \multicolumn{4}{|l|}{ Scrophulariaceae } \\
\hline Eremophila bignoniflora & Emu bush & Australia & $\ldots$ \\
\hline Myoporum laetum & Myoporum & New Zealand & 1 \\
\hline \multicolumn{4}{|l|}{ Solonaceae } \\
\hline Brugmansia 'Charles Grimaldi' & Charles Grimaldi angel's trumpet & Horticultural origin & $\ldots$ \\
\hline Lycianthes rantonnei & Blue potato bush & Argentina, Paraguay & 1 \\
\hline \multicolumn{4}{|l|}{ Taxodiaceae s.s. (Cupressaceae s.1.) } \\
\hline Sequoia sempervirens & Coast redwood & Western North America & $\ldots$ \\
\hline Taxodium mucronatum & Mexican swamp cypress & Mexico & $\ldots$ \\
\hline \multicolumn{4}{|l|}{ Theaceae } \\
\hline Camellia hiemalis 'Kanjiro' & Kanjiro camellia & Horticultural origin & $\ldots$ \\
\hline C. sinensis & Tea plant & Western China & $\ldots$ \\
\hline Gordonia axillaris & $\ldots$ & Southeast Asia & $\ldots$ \\
\hline \multicolumn{4}{|l|}{ Ulmaceae } \\
\hline Ulmus parvifolia & Chinese elm & East Asia & 175 \\
\hline \multicolumn{4}{|l|}{ Verbenaceae } \\
\hline Lippia umbellata (syn. L. torresii) & $\ldots$ & Mexico, Central America & $\ldots$ \\
\hline
\end{tabular}

Table 3. Polyphagous shot hole borer (PSHB)-Fusarium sp. complex collected from tree species found in two botanical gardens located in a heavily infested part of Los Angeles County

\begin{tabular}{|c|c|c|c|}
\hline Symptoms & Number of tree species & Fraction of all species (\%) & Fraction of attacked species (\%) \\
\hline Total number & 335 & $\ldots$ & $\ldots$ \\
\hline Attacked by PSHB & 207 & 62 & $\ldots$ \\
\hline Host for Fusarium sp. & 112 & 33 & 54 \\
\hline Weeping spots on bark & 147 & 44 & 71 \\
\hline Powder depositions & 22 & 7 & 11 \\
\hline Gumming on bark & 69 & 20 & 33 \\
\hline Reproductive hosts for the beetle & 19 & 6 & 9 \\
\hline
\end{tabular}

\section{Results}

Host identification. Of 335 tree species (in 83 families) examined (Tables 1 and 2), 207 (62\%) had symptoms consistent with attack by PSHB (small entry holes in tree bark, wet spots, gumming, white powdery exudate, and frass; Tables 1 and 3; Figs. 3A$\mathrm{K}$ and $4 \mathrm{~A}-\mathrm{C}$ ). The 207 species belonged to 58 families, with origins representing all the continents except Antarctica. Among the attacked species were 11 species native to California, and 13 that are agriculturally important crops in California (see below). There were 128 tree species in 58 families without obvious signs of attack by PSHB (Table 2). Thirty-three plant families examined contained species that were both attacked and not attacked by PSHB.

Fungal DNA was extracted from Fusarium sp. isolates recovered from symptomatic tissues of 113 of the 207 tree species $(54 \%)$ attacked by PSHB. In all cases, sequences of the ITS, EF1- $\alpha$, RPB1, and RPB2 genes of the fungus were identical to the respec- tive known sequences of isolates of Fusarium sp. from Mendel et al. (15) (GenBank accessions JQ038007 to -038034) and Eskalen et al. (7) (GenBank accessions JQ723753 to -723756 and JQ723760 to -723763). These findings indicate the susceptibility of the host tree to the growth of Fusarium sp., at least at the site of the entry hole. Fungal sequences generated in the current study have been deposited in GenBank under the following accession numbers: ITS, JX891673 to -JX891784; EF1- $\alpha$, JX891785 to -JX891896; RPB1, JX891897 to -JX892008; and RPB2, JX892009 to -JX892120. Fusarium sp. was isolated from 113 tree species in 40 families. Families with the highest number of infected species were the Aceraceae, Fabaceae, and Fagaceae.

Of the tree species susceptible to varying degrees of attack by PSHB, 19 were suitable for beetle reproduction. Suitability for reproduction was confirmed by either the presence of eggs, larvae, pupae, or teneral females or the presence of males in the galleries (Table 3; Fig. 5). This number might be an underestimate, because 

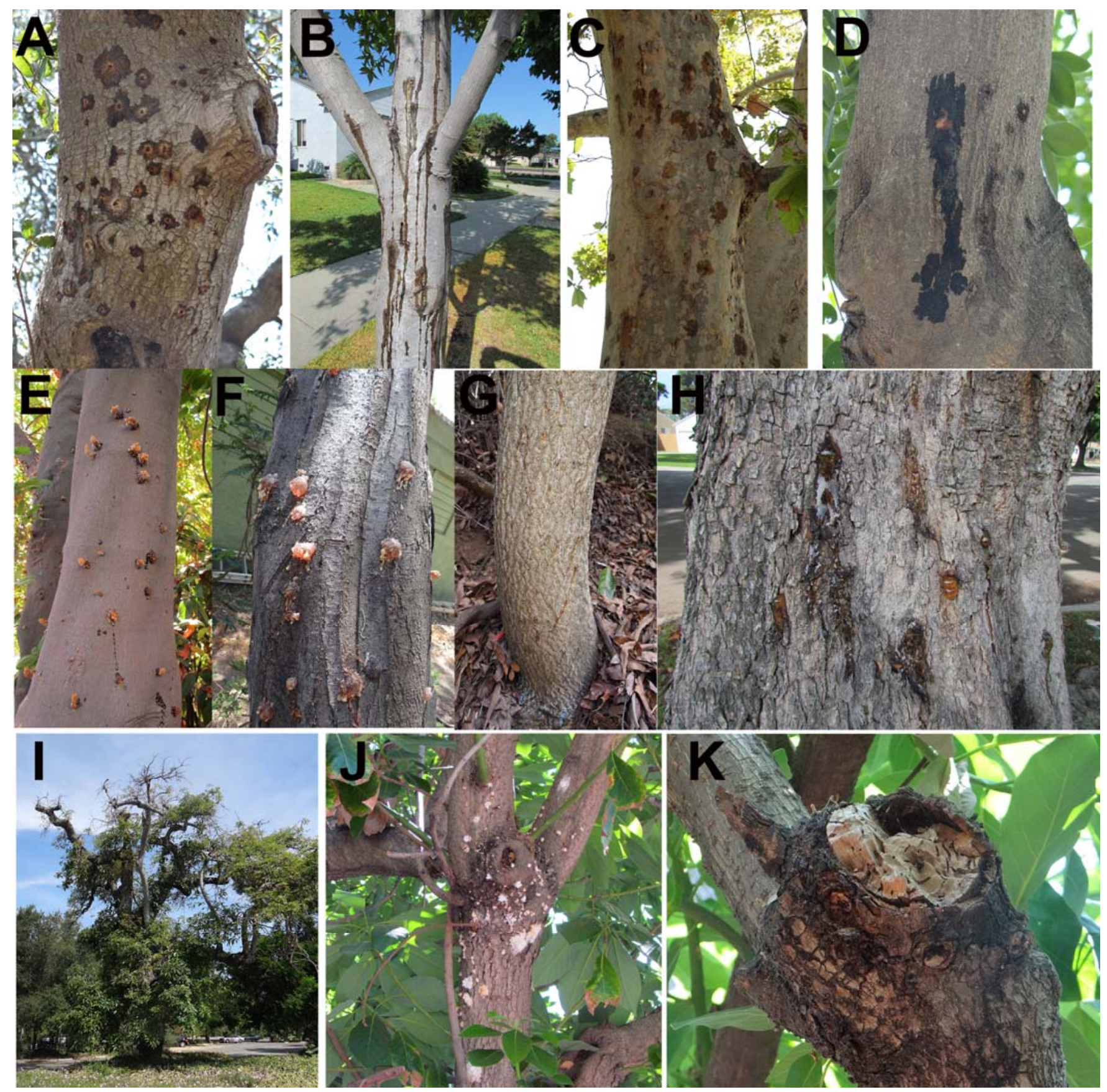

Fig. 3. A-D, External symptoms of staining; E-H, gumming; and I-K, branch dieback for polyphagous shot hole borer (PSHB)-Fusarium dieback disease on A, Quercus agrifolia; B, Liquidambar styraciflua; C, Platanus racemosa; D, Senna specatibilis var. excelsa (syn. Cassia excelsa); E, Alectryon excelsus; F, Acacia melanoxylon; G, Alianthus altissima; H, Koelreuteria elegans; and I-K, Persea americana.

confirmation requires cutting into trees, which was not logistically possible at the time due to the limited number of trees that represent each species within the botanical gardens' collection.

All tree species considered to be reproductive hosts had severe branch dieback (Fig. 3I-K), and the death of mature reproductive hosts that were infested with FD was observed for Quercus robur, Acer negundo, and $R$. communis. A. negundo and $Q$. robur also exhibited leaf wilting and discoloration on the branches prior to dieback and tree death.

Symptom characterization. Symptoms surrounding the beetle entry hole included staining (71\% of hosts), gum deposition (33\% of hosts), and white powder-like exudates (11\% of hosts) (Figs. 3, 4 , and 6 , Tables 1 and 3). Staining was characterized by a moist discoloration of the outer bark that oozed out of the entry holes in various amounts, depending on the host (Fig. 3A-D). Discoloration caused by fungal colonization was observed underneath the inner bark layer when the beetle entry hole penetrated through the cambium and into the xylem (Figs. 7 and 8). On various hosts, PSHB attack resulted in the deposition of an amber colored gum that exuded through the entry holes and varied in consistency between spongy and pliable to solid and hard, depending on the host (Table 1, Fig. 3E-H). After peeling back the outer bark beneath the gum deposition, tissue discoloration was observed and subsequent recovery of Fusarium sp. occurred on $46 \%$ of the tree species showing a gumming response (Table 2). Otherwise, the beetle was found embedded within the gum deposit, and there was no discoloration of the plant tissue. A white powdery exudate was observed on some species (Table 1). On avocado, it characteristically emerged from entry holes and formed a white "sugar volcano" approximately 1 to $15 \mathrm{~cm}$ in diameter and 0.5 to $5 \mathrm{~cm}$ in height (Fig. 4A). Frass was occasionally observed, particularly on trees in the Aceraceae (Fig. 4B). 


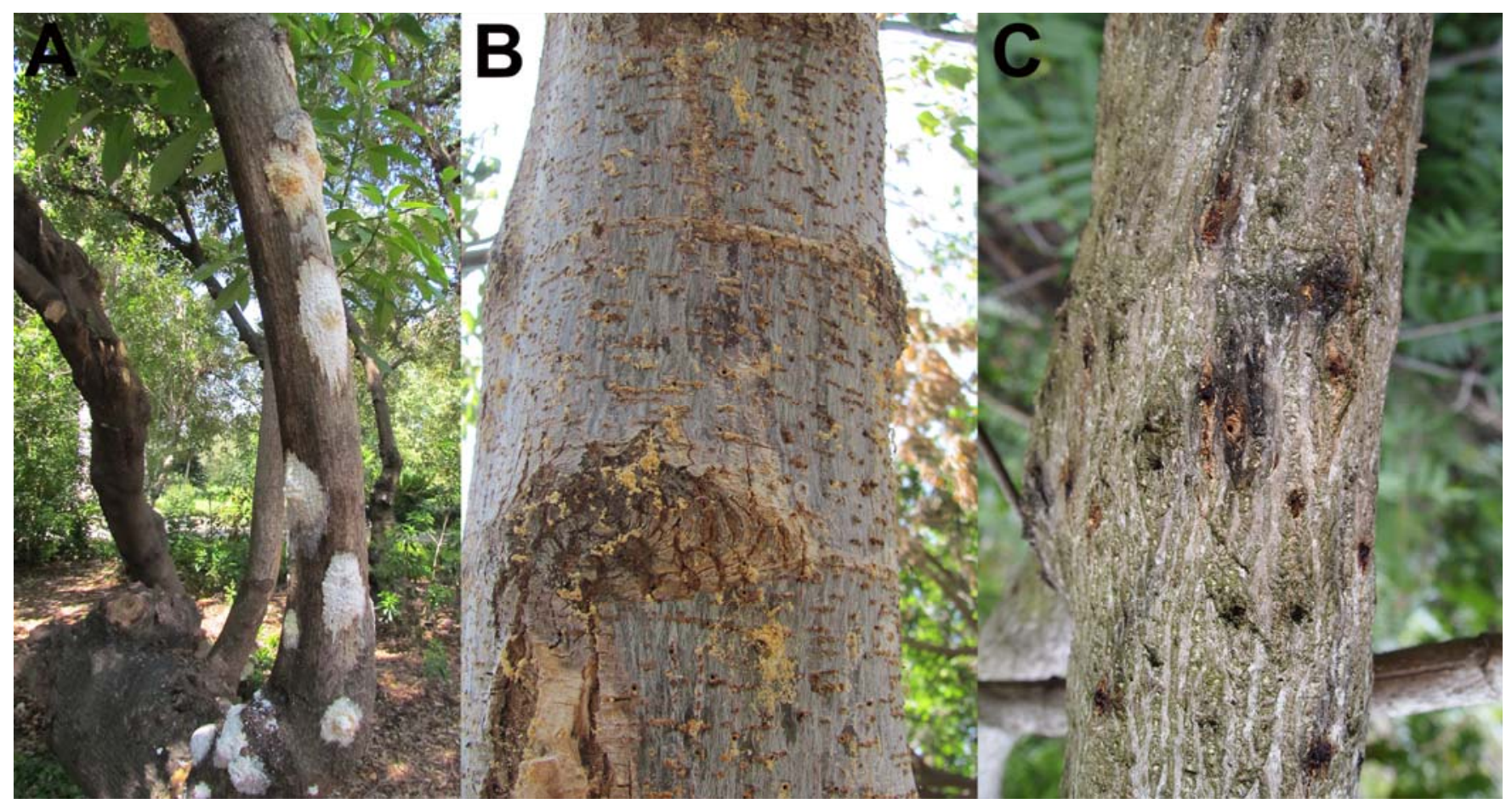

Fig. 4. A, External symptoms of polyphagous shot hole borer and Fusarium dieback disease that are characteristic for Persea americana (white powder exudate/sugar volcano); B, Acer negundo, (abundant entry holes associated with frass); and C, Ricinus communis (staining).

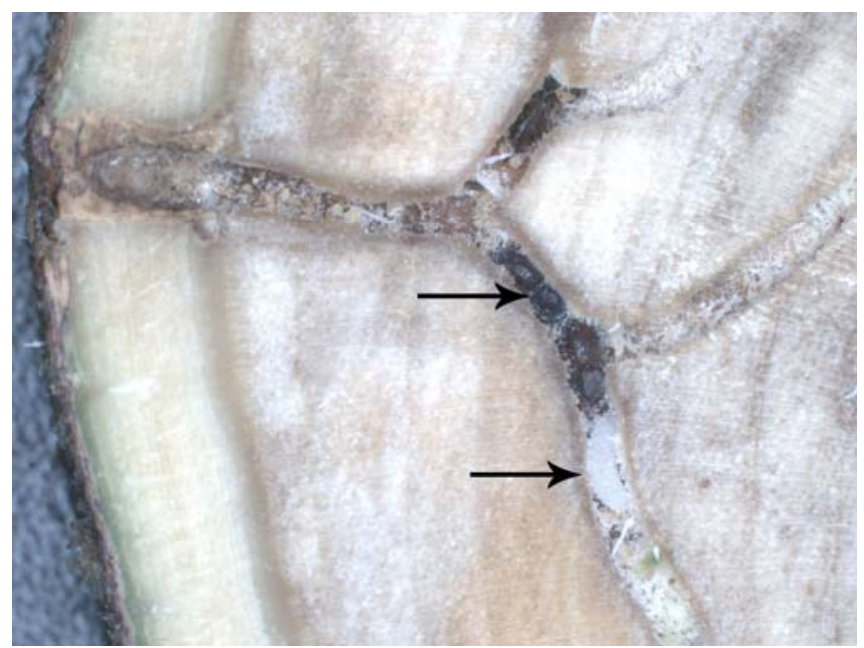

Fig. 5. Offspring (top arrow) and larvae (bottom arrow) in beetle galleries confirm Acer negundo as a reproductive host of polyphagous shot hole borer.

In total, $26 \%$ of the identified hosts of FD had branch dieback on over $50 \%$ of the tree. Symptoms of branch dieback were typically observed on primary branches where areas of weakness caused by beetle and fungal colonization were observed at the branch nodes (Figs. 3I-K and 8A-F). For trees attacked by PSHB, the likelihood of a higher than $50 \%$ branch dieback was positively correlated with presence of Fusarium sp. and increasing severity of PSHB attack, and negatively correlated with symptoms of gumming (Table 4).

Estimated frequency of trees affected by PSHB-Fusarium sp. complex in the urban forest. The botanical garden survey was used to determine the susceptibility of 103 common street tree species (Table 5). In all, 53 species were attacked by PSHB and, of these, Fusarium sp. was isolated from 36 . The beetle was able to reproduce in six of these species. Relating these numbers to the relative abundance of the different trees in the urban environment of southern California (4) reveals that, of the individual street trees, over half $(56 \%)$ belong to species that are attacked by the PSHB,

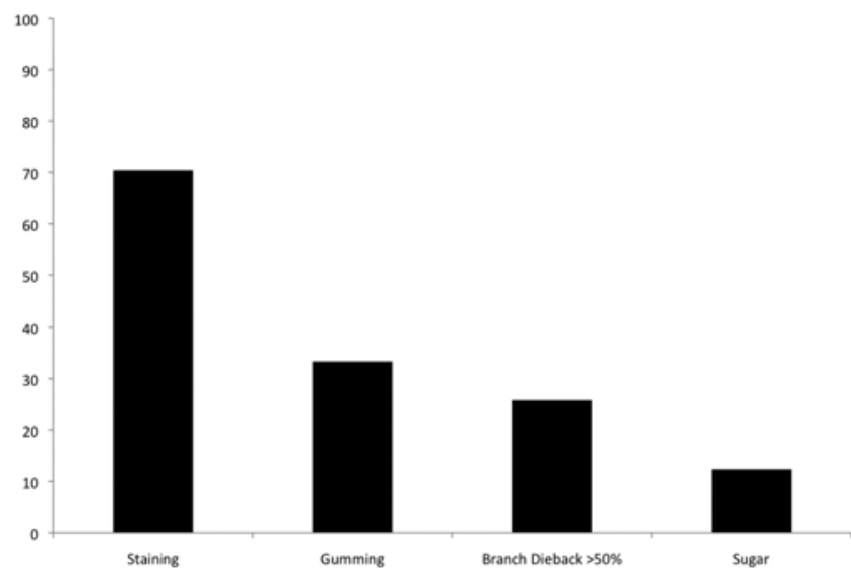

Fig. 6. Proportion of trees prone to polyphagous shot hole borer attack with respective symptoms.

$48 \%$ belong to species that are hosts to the Fusarium sp., and $26 \%$ belong to species that are reproductive hosts of PSHB (Table 5).

FD complex in agriculturally important plants. In this survey, 13 agriculturally important crops displayed signs and symptoms consistent with PSHB attack (Table 1). Of these, growth of the Fusarium sp. was confirmed on avocado, eastern mulberry, olive, macadamia, Turkish hazelnut, loquat, peach, grapevine, and citrus (although the latter does not appear to be commonly attacked). Avocado was the only species confirmed as a reproductive host for PSHB. Symptoms consistent with attack by PSHB were detected on cassava, Japanese persimmon, Japanese crabapple, and eastern black walnut but the Fusarium sp. was not detected growing on these species. The following agricultural crops showed no evidence of PSHB attack: date palm, pomegranate, edible fig, apple, and most citrus tree varieties (see above; Table 2).

\section{Discussion}

Host range, symptoms, and plant responses. In this study, we present the host range of an emerging generalist pest-pathogen complex in California. The behavior of PSHB was consistent with 


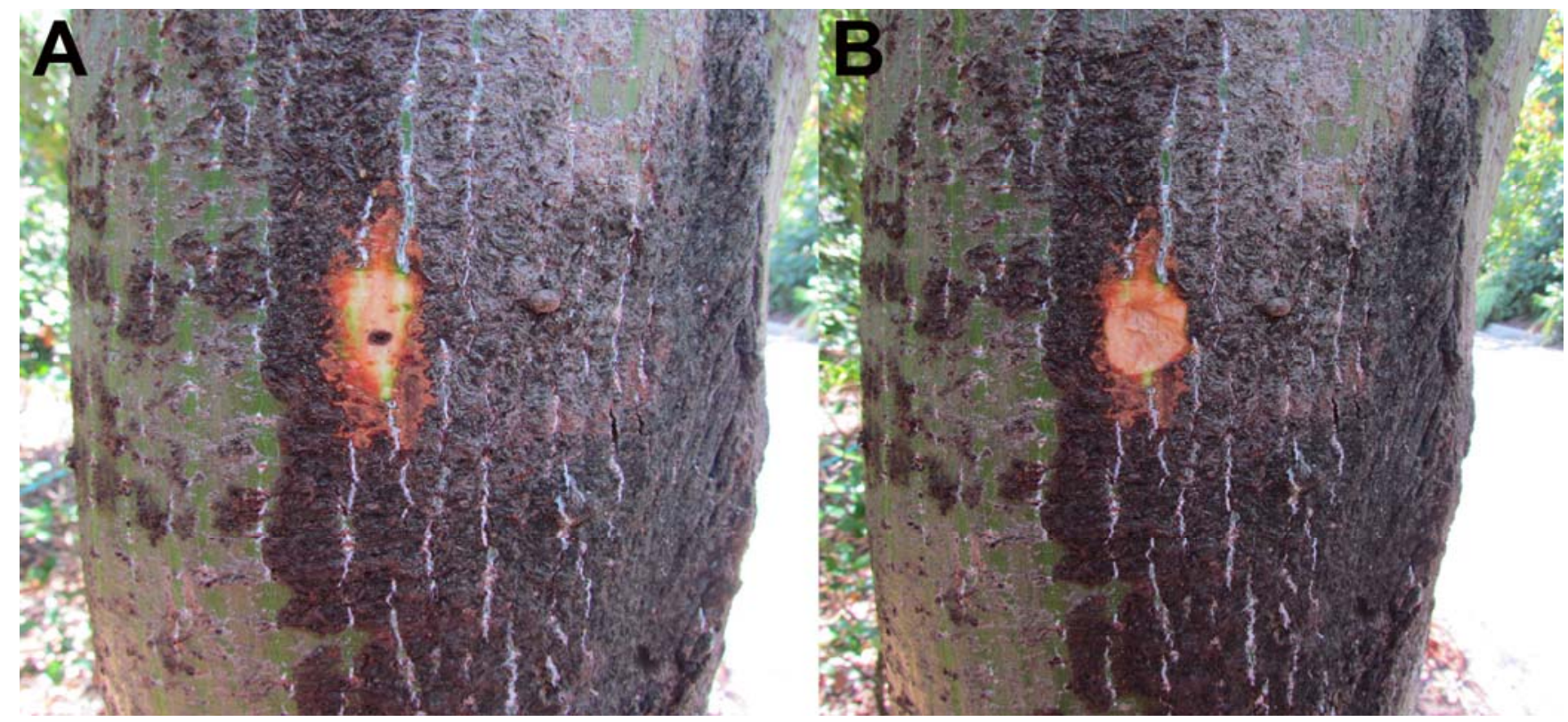

Fig. 7. Evidence of an unsuccessful attempt by polyphagous shot hole borer to establish a gallery in Acer pectinatum subsp. laxiflorum. A, Depth of penetration by the beetle is less than $1 \mathrm{~mm}$. B, No sign of fungal colonization beneath the cambium.

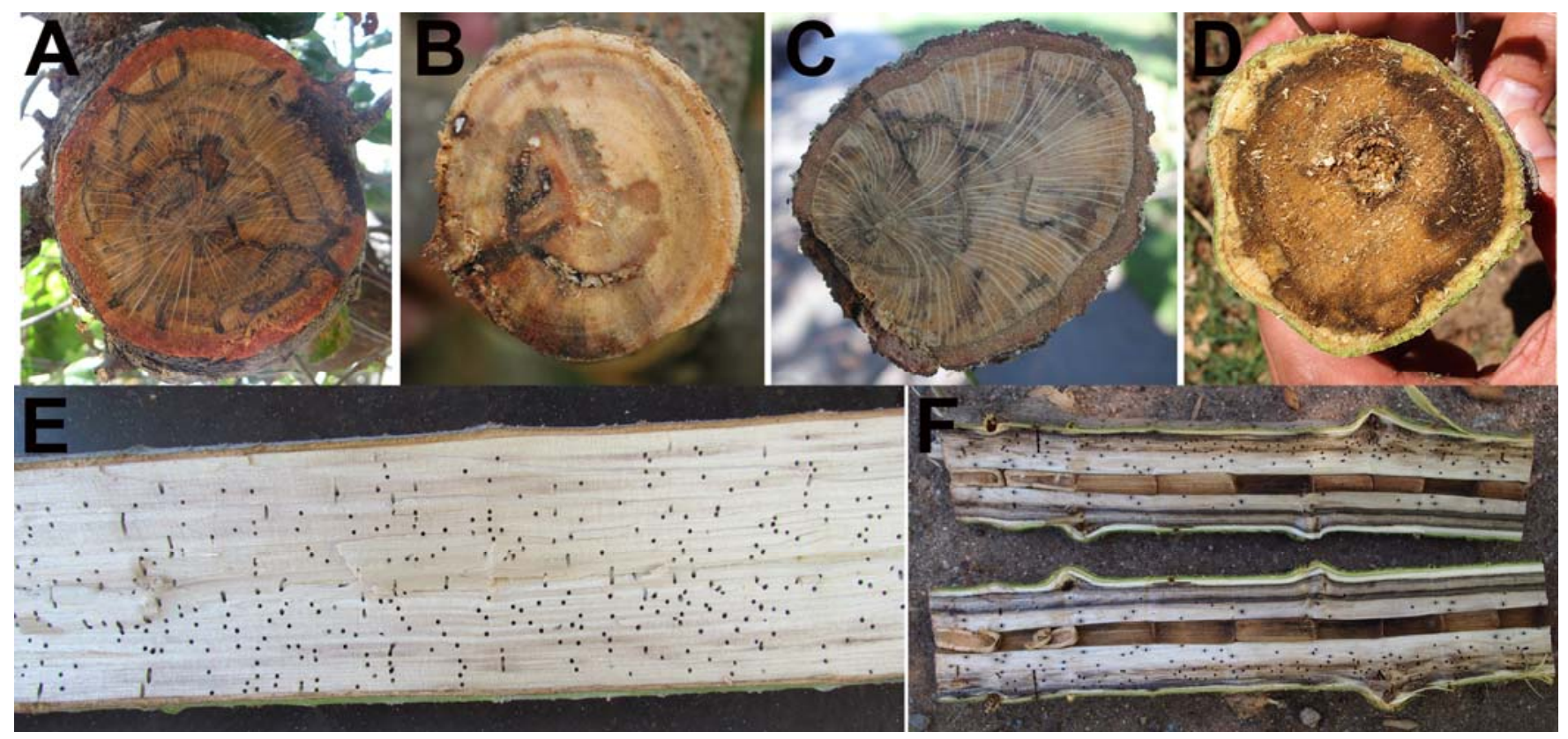

Fig. 8. A, Internal symptoms of polyphagous shot hole borer or Fusarium dieback disease showing gallery formation discoloration caused by fungal colonization on Quercus agrifolia; B, Persea americana; C, Quercus robur, and D, Ricinus communis in cross section; and E, Acer negundo and F, R. communis in longitudinal section.

the ecological group of first-degree primary polyphagous borers that invade healthy trees in many different plant families $(5,12,22,24)$. Plant species attacked by the FD disease complex have worldwide origins, and include species such as the critically endangered dawn redwood (Metasequoia glyptostroboides) from China. In purely economic terms, however, the threat posed by FD to important agricultural crops, ornamental trees used for landscaping, and native forest tree species in California is a major concern. The wide host range of PSHB described in our southern California study was consistent with literature reports for the "tea shot hole borer" (5). However, with the present uncertainty about the species status of individuals morphologically recognized as E. fornicatus, the host range reported by Danthanarayana (5) may actually refer to the hosts of two (or more) cryptic species, each with a more limited host range.

Currently, it is difficult to estimate the longevity of trees infested by either the beetle or fungus alone, or trees that are reproductive hosts. Even though a tree species is not a reproductive host, an attempted PSHB attack may serve as an infection site for Fusarium sp. In some cases, Fusarium sp. was not able to infect the tissue. Even on known hosts of the fungus, tree infection required penetration into at least the cambium layer, which generally varied in depth below the outer bark between species. Given that $82 \%$ of species susceptible to FD in the present study are not reproductive hosts, we conclude that infection is most likely due to susceptibility of the tree to the fungus if the beetle is able to penetrate into or through this critical layer of tissue. Based on the previous observations of Fusarium sp. clogging conductive tissue within the tree $(7,15)$ and dead or dying reproductive hosts infested with FD, and given that there is a correlation between severity of the beetle attack (which thereby increases severity of infection by Fusarium sp.) and the observed dieback, we may predict that, over time, infection by Fusarium sp. may lead to tree death in some species. 
Although gumming was present on some tree species from which Fusarium sp. was also recovered, severe branch dieback was more likely in trees that did not exhibit gumming. This suggests that trees with gumming may be more resistant to beetle (12) and fungal attack. Most trees with gumming did not have necrotic tissues beneath the bark because the gum provided a physical barrier for beetle and fungal entry into the plant. This pattern is consistent with attack to trees by the TSHB in southern Asia, although there is no mention of resistance to fungal infection (5). Host response and resistance to disease or beetle infestation of this particular complex needs to be studied further to control disease establishment and spread.

Potential threat to California landscapes, agriculture, and global plant communities. The research and understanding of the distribution and impact of the FD complex are in the very early stages. However, the ability of this complex to attack multiple tree species in a large number of families suggests that it may become established across a range of plant communities on both a local and global scale. Phytophthora ramorum is an example of a generalist pathogen within California that also has a global distribution. It is the cause of sudden oak death, or ramorum dieback. This pathogen has killed thousands of oaks, tanoaks, and other woody hosts in California plant communities $(6,13,19,21)$. However, the number of families susceptible to natural attack by Fusarium sp. in this study is five times higher than that of P. ramorum (6).

Reproductive hosts are negatively impacted when infected with FD and perpetuate healthy populations of PSHB, which facilitates long-term disease establishment and spread within a system. This study revealed that 5 of the 11 California native plant species surveyed were reproductive hosts of PSHB. This suggests that native plant communities and landscapes with these host plants are in danger of attack. Natural plant communities that are particularly vulnerable in southern California because reproductive hosts of PSHB are dominant members of the plant community include mixed evergreen forests, oak woodlands, foothill woodlands, and riparian habitats. Also noteworthy was that each continent on the globe had at least one reproductive host in this study, excluding Antarctica. This indicates a potential for FD establishment on a global scale. Studies on climatic conditions and other factors that influence PSHB distribution need to be implemented to determine the degree to which areas in other parts of the world are at risk for establishment by PSHB and Fusarium sp.

The juxtaposition of agriculture, urban landscapes, and forests in southern California harboring reproductive host species allows for potential rapid disease spread over a large geographic area that would otherwise be limited to islands of suitable habitat for PSHB and Fusarium sp. As an example, the urban forest in southern Cali-

Table 4. Parameter estimate, odds ratio, and $P$ values for the logistic regression model describing factors contributing to the likelihood of branch dieback $>50 \%$ on trees attacked by the polyphagous shot hole borer (PSHB) (whole model $P>\chi^{2} 0.0035$ and 0.0003 )

\begin{tabular}{lclc}
\hline Variable & Estimate & Odds ratio & $\boldsymbol{P}>\boldsymbol{\chi}^{\mathbf{2}}$ \\
\hline Fusarium sp. & 0.5269 & 2.869 & 0.0065 \\
Gumming & -0.4048 & 2.610 & 0.0202 \\
PSHB severity & 0.9553 & $3.4147-6.9055$ & 0.0004 \\
\hline
\end{tabular}

Table 5. Number of tree species and abundance of tree specimens susceptible to the polyphagous shot hole borer (PSHB)-Fusarium sp. complex in the southern Californian urban forest ${ }^{\mathrm{a}}$

\begin{tabular}{lcc}
\hline Variable & $\begin{array}{c}\text { Number of } \\
\text { species }\end{array}$ & $\begin{array}{c}\text { Abundance } \\
(\boldsymbol{\%})^{\mathbf{b}}\end{array}$ \\
\hline Total number & 103 & $\ldots$ \\
Attacked by PSHB & 53 & 56 \\
Host for Fusarium sp. & 36 & 48 \\
Reproductive hosts for the beetle & 6 & 26 \\
\hline
\end{tabular}

${ }^{a}$ Estimations were made using a representative tree species list (4).

${ }^{b}$ Percentage of all specimens in the urban forest. fornia could exacerbate the spread of FD, given that $48 \%$ of the street trees in southern California (4) belong to species that were confirmed as susceptible to FD. Six of these tree species are also reproductive hosts for PSHB, including American sweetgum, which is one of the most commonly planted street trees. Additionally, castor bean, a reproductive host and weed with a global distribution, is prevalent in urban, agriculturally important, and riparian areas and in the interface between these landscapes in southern California (27). The potential costs associated with the infestation of the urban forest may become substantial.

The FD complex is of special concern to the California avocado industry. It is already established and causing significant losses in commercial avocado groves in Israel (15). Given the high frequency and broad distribution of reproductive hosts and other host species throughout the region, disease spread and widespread establishment into commercial avocado groves in southern California is almost inevitable. At the time of writing, symptoms of FD have not been observed in commercial citrus groves or vineyards but have been confirmed in two commercial avocado groves in Los Angeles County. Disease distribution throughout southern California is currently being investigated.

Although studies are underway to determine host resistance, disease distribution, and impact, the results from this study suggest that plants confirmed as nonhosts, or hosts that display symptoms of resistance to PSHB, be given priority in future landscape plantings.

\section{Acknowledgments}

We thank the California Avocado Commission for supporting this research; Huntington Library, Art Collections, and Botanical Gardens and the Los Angeles County Arboretum for allowing us to conduct the survey for this study; P. Meyers from the Huntington herbarium for assistance with editing the scientific names of host and nonhost species; F. McDonogh for assistance with surveying the LA Arboretum; J. Mayorquin, D. Wang, L. Yeung, and F. Na (Department of Plant Pathology and Microbiology, University of California, Riverside) for assistance with sample processing and molecular identification of fungi; A. Coelho, Jr. (Department of Entomology, University of California, Riverside) for assistance with sample collection; R. Hofshi and M. L. Arpaia for ongoing support in this research; and the anonymous reviewers of this manuscript for comments and suggestions.

\section{Literature Cited}

1. Benson, D. A., Karsch-Mizrachi, I, Lipman D. J., Ostell J., and Wheeler D. L. 2008. GenBank. Nucleic Acids Res 36:D25-D30.

2. Cenis, J. L. 1992. Rapid extraction of fungal DNA for PCR amplification Nucleic Acids Res. 20:2380

3. Chang, D. C., G. B. Grant, K. O'Donnell, K. A. Wannemuehler, J. NobleWang, C. Y. Rao, L. M. Jacobson, C. S. Crowell, R. S. Sneed, F. M. T. Lewis, J. K. Schaffzin, M. A. Kainer, C. A. Genese, E. C. Alfonso, D. B. Jones, A. Srinivasan, S. K. Fridkin, and B. J. Park. 2006. A multistate outbreak of Fusarium keratitis associated with use of a new contact lens solution. JAMA 296:953-963.

4. City of Orange Species Frequency Report with Characteristics website 1999. http://www.cityoforange.org/civicax/filebank/blobdload.aspx?blobid $=3499$

5. Danthanarayana, W. 1968. The distribution and host-range of the shot-hole borer (Xyleborus fornicatus Eichh.) of tea. Tea Q. 39:61-69.

6. Davidson, J. M., Werres, S., Garbelotto, M., Hansen, E. M., and Rizzo, D. M. 2003. Sudden oak death and associated diseases caused by Phytophthora ramorum. Plant Health Prog. Online publication. doi:10.1094/ PHP-2003-0707-01-DG

7. Eskalen, A., Gonzalez, A., Wang, D. H., Twizeyimana, M., Mayorquin, M., and Lynch, S. C. 2012. First report of Fusarium sp. and its vector tea shot hole borer (Euwallacea fornicatus) causing Fusarium dieback on avocado in California. Plant Dis. 96:1070.

8. Folmer, O., Black, M., Hoeh, W., Lutz, R., and Vrijenhoek, R. 1994. DNA primers for amplification of mitochondrial cytochrome $\mathrm{C}$ oxidase subunit I from diverse metazoan invertebrates. Mol. Marine Biol. Biotechnol. 3:294299.

9. Hall, T. A. 1999. BioEdit: a user friendly biological sequence alignment and analysis program from Windows 95/98/NT. Nucleic Acids Symp. 41:95-98.

10. Hebert, P. D. N., Cywinska, A., Ball, S. L., and deWard, J. R. 2003. Biological identifications through DNA barcodes. Proc. R. Soc. Lond. B 270:313-321.

11. Hulcr, J., Mann, R., and Lukazs, L. S. 2011. The scent of a partner: ambrosia beetles are attracted to volatiles from their fungal symbionts. J. Chem. Ecol. 37:1374-1377.

12. Kovach, J., and Gorsuch, C. S. 1985. Survey of ambrosia beetle species' 
infesting South Carolina peach orchards and a taxonomic key for the most common species. Agric. Entomol. 2:238-247.

13. Maloney, P. E., Lynch, S. C., Kane, S. F., Jensen, C. E., and Rizzo, D. M. 2005. Establishment of an emerging generalist pathogen in redwood forest communities. J. Ecol. 93:899-905.

14. McDonald, V., and Eskalen, A. 2011. Botryosphaeriaceae species associated with avocado branch cankers in California. Plant Dis. 95:1465-1473.

15. Mendel, Z., Protosav, A., Sharon, M., Zveibil, A., Ben Yahuda, S., O’Donnell, K., Rabaglia, R., Wysoki, M., and Freeman, S. 2012. An Asian ambrosia beetle Euwallacea fornicatus and its novel symbiotic fungus Fusarium sp. pose a serious threat to Israeli avocado industry. Phytoparasitica, Online publication. doi:10.1007/s12600-012-0223-7

16. O’Donnell, K., Sarver, B. A. J., Brandt, M., Chang, D. C., Noble-Wang, J., Park, B. J., Sutton, D. A., Benjamin, L., Lindsley, M., Padhye, A., Geiser, D. M., and Ward, T. J. 2007. Phylogenetic diversity and microsphere arraybased genotyping of human pathogenic fusaria, including isolates from the multistate contact lens-associated U.S. keratitis outbreaks of 2005 and 2006. J. Clin. Microbiol. 45:2235-2248.

17. O'Donnell, K., Cigelnik, E., and Nirenberg, H. 1998. Molecular systematics and phylogeography of the Gibberella fujikuroi species complex. Mycologia 90:465-493.

18. O’Donnnell, K., Sutton, D. A., Rinaldi, M. G., Sarver, B. A. J., Arunmozhi Balajee, S., Schroer, H., Summerbell, R. C., Robert, V. A. R. G., Crous, P. W., Zhang, N., Aoki, T., Jung, K., Park, J., Lee, Y., Kang, S., Park, B., and Geiser, D. M. 2010. Internet-accessible DNA sequence database for identifying Fusaria from human and animal infections. J. Clin. Microbiol. 48:3708-3718

19. Parke, J. L., and Lucas, S. 2008. Sudden oak death and ramorum blight. Plant Health Instructor. Online publication. doi:10.1094/PHI-I-2008-0227-01

20. Rabaglia, R. J., Dole, S. A., and Cognato, A., 2006. Review of American
Xyleborina (Coleoptera: Curculioniadae: Scolytinae) occurring north of Mexico, with an illustrated key. Ann. Entomol. Soc. Am. 99:1034-1055.

21. Rizzo, D. M., and Garbelotto, M. 2003. Sudden oak death: endangering California and Oregon forest ecosystems. Front. Ecol. Environ. 1:197-204.

22. Rudinsky, J. A. 1962. Ecology of Scolytidae. A. Rev. Ent. 7:327-348.

23. Rugman-Jones, P. S., Hoddle, M. S., Amrich, R., Heraty, J. M. StouthamerIngel, C. E., and Stouthamer, R. 2012. Phylogeographic structure, outbreeding depression, and reluctant virgin oviposition in the bean trips, Caliothrips fasciatus (Pergande) (Thysanoptera: Thripidae), in California. Bull. Entomol. Res. Online publication. doi:10.1017/S0007485312000302

24. Schedl, K. E. 1958. Breeding habits of Arboricole insects in central Africa. Pages 183-197 in: Proc. Int. Cong. Ent. 10th Meet. Montreal, Quebec, Canada.

25. Slippers, B., Crous, P. W., Denman, S., Coutinho, T. A., Wingfield, B. D., and Wingfield, M. J. 2004. Combined multiple gene genealogies and phenotypic characters differentiate several species previously identified as Botryosphaeria dothidea. Mycologia 96:83-101.

26. Song, H., Buhay, J. E., Whiting, M. F., and Crandall, K. A. 2008. Many species in one: DNA barcoding overestimates the number of species when nuclear mitochondrial pseudogenes are coamplified. Proc. Natl. Acad. Sci. USA 105:13486-13491.

27. United States Department of Agriculture, Natural Resources Conservation Service. 2012. Plant Profile website. http://plants.usda.gov/java/profile? symbol=RICO3

28. Wood, S. L. 1989. Nomenclatural changes and new species of Scolytidae (Coleoptera), Part IV. Great Basin Nat. 49:167-185.

29. Zhang, N., O'Donnell, K., Sutton, D. A., Nalim, F. A., Summerbell, R. C., Padhye, A. A., and Geiser, D. M. 2006. Members of the Fusarium solan species complex that cause infections in both humans and plants are common in the environment. J. Clin. Microbiol. 44:2186-2190. 